\title{
Effects of Tube Processing on the Fatigue Life of Nitinol
}

\author{
Paul Adler $^{1,2} \cdot$ Rudolf Frei $^{3} \cdot$ Michael Kimiecik $^{4} \cdot$ Paul Briant $^{4} \cdot$ Brad James $^{4} \cdot$ \\ Chuan Liu $^{2}$
}

Published online: 20 February 2018

(C) The Author(s) 2018. This article is an open access publication

\begin{abstract}
Nitinol tubes were manufactured from Standard Grade VIM-VAR ingots using Tube Manufacturing method "TM-1." Diamond-shaped samples were laser cut, shape set, then fatigued at $37{ }^{\circ} \mathrm{C}$ to $10^{7}$ cycles. The 50,5 , and $1 \%$ probabilities of fracture were calculated as a function of number of cycles to fracture and compared with probabilities determined for fatigue data published by Robertson et al. (J Mech Behav Biomater 51:119-131, 2015). Robertson tested similar diamonds made from the same standard grade of Nitinol as in the current study, two other standard grades of Nitinol, and two high-purity grades of Nitinol expressly designed to improve fatigue life. Robertson's tubes were manufactured using Tube Manufacturing method "TM-2." Fatigue performance of TM-1 and TM-2 diamonds were compared: At $10^{7}$ cycles, strain amplitudes corresponding to the three probabilities of fracture of the TM-1 diamonds were 2-3 times those of the TM-2 diamonds made from the same grade of Nitinol, and comparable to TM-2 diamonds made from the higherpurity materials. This difference is likely a result of the differences in tube manufacturing techniques and effects on resulting microstructures. Microstructural analyses of
\end{abstract}

This article is an invited paper selected from presentations at the International Conference on Shape Memory and Superelastic

Technologies 2017, held May 15-19, 2017, in San Diego, California, and has been expanded from the original presentation.

Paul Adler

paul.adler@northwestern.edu

Invariant-Plane Solutions, LLC, Long Grove, IL, USA

2 Northwestern University, Evanston, IL, USA

3 Vascotube $\mathrm{GmbH}$, Birkenfeld, Germany

4 Exponent, Inc., Menlo Park, CA, USA samples revealed a correlation between the median probability of fracture and median inclusion diameter that follows an inverse power-law function of the form $y \approx x^{-1}$.

Keywords Superelasticity · Fatigue · Nitinol - Inclusion · Medical device $\cdot$ Probability functions

\section{Introduction}

High-cycle fatigue life of Nitinol remains of interest as this unique material is used in an increasing number of Class 3 medical devices as well as for a resurgence of actuator applications [2-5]. With medical devices approved for more demanding cardiovascular applications such as transcatheter aortic and mitral valve repair (TAVR/TMVR), as well as younger cohorts of patients with concomitantly increased required duty cycles, long-term structural integrity of the substrate remains of paramount concern. With these considerations in mind, much attention has been paid to factors affecting the fatigue life of Nitinol to ensure long-term device efficacy. Initial fatigue studies [1, 6-8] using diamond samples meant to represent the fundamental construct of laser-cut devices and capture clinically relevant loading conditions, showed high-cycle fatigue strain limits on the order of $0.4-0.8 \%$. As often occurs in wrought ductile structural alloys, non-metallic inclusions in the form of stringers resulting from axisymmetric working operations act as crack nucleation sites during high-cycle fatigue of Nitinol components. Recent efforts on the part of raw material producers to improve material cleanliness have resulted in notable increases in fatigue life [1,9]. Despite these efforts, however, there has been little published work exploring the effects of metal processing techniques on fatigue properties. 
The current effort compares the effects of two tube processing methodologies, TM-1 and TM-2, on the fatigue life of surrogate samples deemed representative of the fundamental closed cell comprising a self-expanding stent or heart valve. Ideally, such a study would involve manufacturing tubing from the same starting heat of material to minimize effects related to initial ingot cleanliness, composition, etc., while systematically varying tube processing parameters. That was not done in the current effort as tube manufacturing processes at the related companies are considered intellectual property. However, as a result of the thorough and meticulous published work as delineated below [1], it is possible to compare the effects of TM-1 and TM-2 tube processing on fatigue life within the confines outlined herein. The resulting fatigue behaviors are correlated with statistical analyses of microstructures of these alloys and discussed in terms of traditional metallurgical tenets and novel computational modeling of the potency of primary inclusions in fatigue crack nucleation.

\section{Materials, Processes, and Experimental Procedures}

Superelastic (SE) Nitinol can be a notoriously difficult material to consistently draw into tubes with sufficient quality for laser cutting of Class 3 cardiovascular implants. For this reason, specific manufacturing processes can vary significantly among tube redraw producers and are often held as intellectual property (IP). In the case of the tube manufacturing processes used in the current study, such IP prevents full disclosure of all processing steps. However, some differences between manufacturing techniques are known and can be discussed [10].

\section{General Manufacture of Nitinol}

Nitinol ingots weighing from a few hundred to thousands of kilograms are produced at any of a number of mills typically using vacuum-induction melting (VIM), vacuum arc re-melting (VAR), or a combination of these casting techniques. ASTM Standard F2063 [11] defines chemical and trace element requirements. After melting, the Nitinol ingot is forged and rolled at elevated temperatures into a bar or slab. The workpiece is further subject to a series of controlled reductions followed by heating to recrystallize and anneal the matrix necessary for continued working. Round bars with outer diameter of approximately $25 \mathrm{~mm}$ or smaller typically comprise a "mill product" as defined in the ASTM specifications. Round bars are further reduced to smaller diameters for wire products. In the case of lasercut platforms for medical devices or hypotubing, centerless ground round bars are gun drilled or trepanned to introduce a hole down the center of the bar typically at an outer diameter (OD) of approximately $25 \mathrm{~mm}$ or smaller depending on customer requirements. At this point, the workpiece is usually termed a "hollow." Hollows often exhibit $99+\%$ concentricity. Hollows are shipped to redraw houses for further reductions to customer specified final tubing. There are a number of companies that perform final tube drawing operations.

As with tube manufacture in general, successful fabrication of as-received Nitinol tube hollows requires maintaining control of the outer diameter-to-wall thickness ratio during reduction operations. In the case of drawing, this is typically achieved through optimization of die size, design, and materials, as well as, lubricants, mandrel size, mandrel materials, and reduction schedules. Often Nitinol tube manufacturing techniques [12] employ continuous operations in which tubing is wrapped around a bull block, or take-up spool, to reduce equipment set-up costs. While providing cost advantages, the asymmetric stress and strain gradients introduced in the tube during such non-linear drawing operations may have a significant effect on tube uniformity, concentricity, and straightness of the final product, and resulting final device yields. Such rotational drawing operations may also result in asymmetric microstructures and non-uniform mechanical properties. As well, mechanical properties of the mandrel used to control ID have a significant impact on final tube properties. Depending on specific parameters, sinking operations in which tubes are drawn with no mandrel may result in the onset of an 'orange peel' effect on the ID surface. This type of drawing operation is the most economical, especially on thick-walled tubes and tubes smaller than $12 \mathrm{~mm}$ in diameter [13] but does not produce the best surface finish. As the tube thickness changes the surface finish quality decreases. This process is often used in the production of tubing for low-cost lawn furniture [14]. Mandrels with hardness less than that of the Nitinol, termed 'soft mandrels,' are often used as these allow some control over wall thickness while allowing the tube to be drawn multiple times before needing to remove the mandrel. This again provides significant cost advantages especially in the production of very small diameter hypotubing. As with sinking, however, ID surfaces are often severely marred during de-coring (mandrel removal) operations, and wall tolerances suffer decreasing final tube concentricity which can reduce laser-cut component yields. Optimal control of OD and wall thickness is obtained using a hard mandrel where the mandrel hardness exceeds that of the Nitinol tube. Hard mandrels also have the advantage of maintaining a relatively smooth ID surface. The disadvantage of hard mandrel operations is that the mandrel must be removed after each drawing operation increasing the cost of manufacture. 
Many redraw operations use tungsten carbide drawing dies especially during initial (large diameter) reductions again as cost saving measures. In contrast to tungsten carbide, polycrystalline dies (PCD), while very expensive, are extremely lubricious with the very fine diamond grain structure providing a relatively defect-free and very smooth uniform surface. Although most Nitinol tubing used for laser-cut medical devices is centerless ground as part of final fabrication, use of tungsten carbide dies, oxidizing environments during interpass anneals, and abrasive surface cleaning techniques such as sand or grit blasting necessary for removal of oxidized surfaces even solely at large diameters can introduce potent defects that may be maintained and promulgated through the tube thickness affecting final device characteristics.

The redraw company will process the as-received hollows to a size (OD and wall thickness) often termed $A_{\mathrm{o}}$ defined by the cross-sectional area of the workpiece. At $A_{\mathrm{o}}$, the workpiece is given a last full anneal. As a point of interest, under an applied load, the fully annealed Nitinol will undergo non-thermoelastic phase transformation similar to that in a TRIP (TRansformation-Induced Plasticity) steel. In the current study, approximately $30 \%$ cold work (by area reduction) was introduced into the annealed workpiece to achieve the desired thermoelastic or pseudoelastic properties necessary for proper self-expanding device operation at the desired final tube size, $A_{\text {final }}$. Final drawing from $A_{\mathrm{o}}$ to $A_{\text {final }}$ is usually performed in a series of discrete operations in which tubes are drawn straight using either a hard or soft mandrel although sinking may be used as well. At $A_{\text {final }}$, the tubing is pulled under a tensile load through strand anneal equipment for straightening. This final aging treatment imparts the classical 'flag'-shaped superelastic behavior. Final tubing is often centerless ground to customer specified dimensions.

Although starting ingot charge chemistries and cleanliness certainly affect fatigue properties of a final device, it is primarily the effects of this last series of tube reductionsfrom starting hollow through final tubing-on device fatigue life that are explored in the current work.

\section{Tube Manufacturing Process TM-1}

Tube manufacturing method TM-1 is the standard process used by Vascotube GmbH, Birkenfeld, Germany, to produce Nitinol tubing for laser cutting of medical devices from a starting hollow. In the current study, 25.4-mm-outer diameter (OD) hollows were produced from two Standard Grade VIM-VAR Nitinol ingots by SAES Getters S.p.A. Incoming hollows were then processed to a final size of $10 \times 0.457 \mathrm{~mm}(\mathrm{OD} \times$ wall thickness $)$ using tube manufacturing method TM-1. The compositions, transformation temperatures, and microstructural characteristics of the two Standard Grade VIM-VAR Nitinol ingots used in the current research as supplied by the mill, are provided in the columns in Table 1 labeled Ingot 1 and Ingot 2. Using TM-1 processing, two lots of finished tubes were manufactured from Ingot 1 material and one lot from Ingot 2 as described in Table 1. Henceforth, these three lots of final tubing are termed $1-1,1-2$, and $2-1$, respectively.

From the starting 25.4-mm-diameter hollow, all TM-1 reductions employ polycrystalline diamond (PCD) dies, straight single-draw operations using hard mandrels to preserve the high concentricity, and surface and internal qualities of incoming hollows through final tubing. Interpass anneals are performed solely in inert or vacuum environments to minimize surface and internal oxidation that can be detrimental to final tubing properties. Surface cleaning operations ensure high-quality surfaces are maintained throughout processing. Finished tube lots $1-1$, $1-2$, and 2-1 were laser cut into diamond-shaped samples then expanded using shape set parameters typical for a medical device to achieve microstructures representative thereof. Specific parameters are considered IP. Figure 1 shows the final diamond shape as well as high-stress location on the strut similar to that in an in vivo operating device. Finished diamond samples exhibited an $A_{\mathrm{f}}=20 \pm 3{ }^{\circ} \mathrm{C}$ as determined by the bend and free recovery method defined in ASTM F2082-16 [15]. Diamonds were electropolished to remove surface defects and obtain a surface similar to a typical SE Nitinol device implant. Specifics of this process are deemed intellectual property but act to remove approximately $25 \%$ of the material by weight. Cross-sectional optical metallography on processed samples showed no remnant heat-affected zones from laser cutting.

\section{Tube Manufacturing Process TM-2}

Robertson et al. [1] tested diamond surrogates similar to those used in the current study made from three standard grade Nitinol materials and two high-purity grade materials specifically to examine the effects of material cleanliness on fatigue life. The compositions, transformation temperatures, and microstructural characteristics for the Standard Grade VAR, Standard Grade VIM-VAR, and Standard Grade VIM materials supplied by the mills are listed in Table 1 as Ingots A, B, and C, respectively. Similar data for the two high-purity grades of Nitinol also tested in the previous study [1] are labeled as process optimized (PO) VIM-VAR, and high-purity (HP) VAR also listed in Table 1 as Ingots D and E, respectively. As cited in Ref. [1], the term 'Standard' refers to traditional melting practices used in the production of Nitinol using nominal purity raw materials. High purity (HP) refers to the use of ultrahigh-purity raw materials in the VAR melting process. 
Table 1 Chemical composition, transformation temperatures, and microstructural attributes as reported by the mill product suppliers for the current work and the previous study [1]

\begin{tabular}{|c|c|c|c|c|c|c|c|}
\hline \multicolumn{8}{|c|}{ Mill product certification of compliance data } \\
\hline \multirow[t]{3}{*}{ Element, w/o } & \multirow{2}{*}{\multicolumn{2}{|c|}{$\frac{\text { Current study }}{\text { Standard VIM-VAR }}$}} & \multicolumn{5}{|c|}{ Robertson et al. [1] study } \\
\hline & & & \multirow{2}{*}{$\begin{array}{l}\text { Standard } \\
\text { VAR } \\
\text { Ingot A }\end{array}$} & \multirow{2}{*}{$\begin{array}{l}\text { Standard VIM- } \\
\text { VAR } \\
\text { Ingot B }\end{array}$} & \multirow{2}{*}{$\begin{array}{l}\text { Standard } \\
\text { VIM } \\
\text { Ingot C }\end{array}$} & \multirow{2}{*}{$\begin{array}{l}\text { Process optimized } \\
\text { VIM-VAR } \\
\text { Ingot D }\end{array}$} & \multirow{2}{*}{$\begin{array}{l}\text { High-purity } \\
\text { VAR } \\
\text { Ingot E }\end{array}$} \\
\hline & Ingot 1 & Ingot 2 & & & & & \\
\hline Nickel & 55.90 & 55.93 & 55.83 & 55.89 & 55.8 & 55.98 & 56.1 \\
\hline Titanium & Balance & Balance & Balance & Balance & Balance & Balance & Balance \\
\hline $\mathrm{C}$ & 0.0272 & 0.0283 & 0.0020 & 0.0268 & 0.0330 & 0.0269 & 0.0020 \\
\hline $\mathrm{Cr}$ & 0.0004 & 0.0002 & 0.0050 & $<0.0100$ & 0.004 & 0.0100 & 0.0050 \\
\hline Co & 0.0009 & 0.0009 & 0.0050 & $<0.0100$ & 0.003 & 0.0100 & 0.03 \\
\hline $\mathrm{Cu}$ & 0.0004 & 0.0004 & 0.0050 & $<0.0100$ & 0.0010 & 0.0100 & 0.0050 \\
\hline $\mathrm{H}$ & $<0.0050$ & $<0.0050$ & 0.0015 & 0.0008 & 0.0011 & 0.0007 & 0.0008 \\
\hline $\mathrm{Fe}$ & 0.0068 & 0.0066 & 0.0050 & 0.0062 & 0.011 & 0.011 & 0.0063 \\
\hline $\mathrm{Nb}$ & 0.0001 & 0.0001 & 0.0050 & $<0.0100$ & 0.0010 & 0.0100 & 0.0050 \\
\hline $\mathrm{N}$ & 0.0005 & 0.0012 & NR & NR & NR & NR & NR \\
\hline 0 & 0.0152 & 0.0238 & NR & NR & NR & NR & NR \\
\hline $\mathrm{N}+\mathrm{O}$ & 0.0157 & 0.0250 & 0.0274 & 0.0197 & 0.041 & 0.026 & 0.0063 \\
\hline $\begin{array}{l}\text { Transformation temperature*, } \\
A_{\mathrm{f}},{ }^{\circ} \mathrm{C}\end{array}$ & -4 & -3 & -19 & -10 & -1 & -9 & -9 \\
\hline Maximum NMI length, $\mu \mathrm{m}$ & 9 & 15 & 38 & 17 & 7 & 19 & 15 \\
\hline Area fraction, $\%$ & 0.74 & 0.75 & 1.25 & 1.1 & 0.7 & 1.18 & 0.46 \\
\hline
\end{tabular}

Values not reported are designated "NR"

*Measured by differential scanning calorimetry (DSC) in accordance with ASTM F2004 [24]
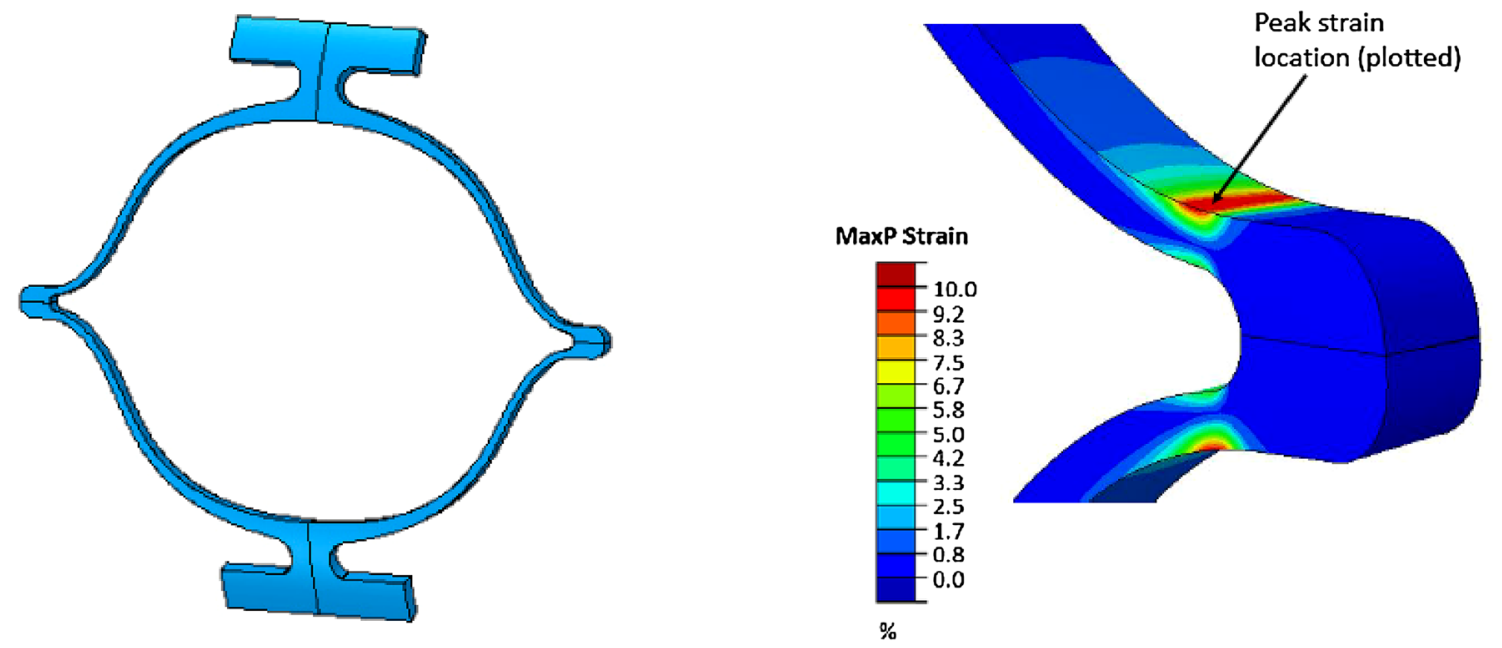

Fig. 1 Diamond sample used for fatigue testing (left image). Finite element model used to compute strain-displacement relations for fatigue testing of diamond surrogate samples (right image)

Process optimized (PO) refers to melting practices specifically designed to reduce average non-metallic inclusion size. A grade of exceptionally clean Nitinol termed "highcycle fatigue" (HCF), not tested, will soon be available [9]. The cleaner and optimized grades of material are designed specifically to improve fatigue life of components.
In the previous study [1], five hollows ranging in starting diameter from 10.5 to $25.4 \mathrm{~mm}$ were investigated. Hollows were obtained from a variety of mill product suppliers and processed to $8 \times 0.37 \mathrm{~mm}(\mathrm{OD} \times$ wall thickness $)$ using tube manufacturing process TM-2 at G. Rau, GmbH, Pforzheim, Germany. All tubes received the identical final drawing sequence specifically to minimize effects of tube 
manufacturing on fatigue properties. As in the current work, Robertson imparted $30 \%$ cold work during reductions from $A_{\mathrm{o}}$ to $A_{\text {final }}$. Tubes were then straightened in a horizontal furnace. Tube process parameters were chosen to achieve as close to identical superelastic mechanical properties as possible (upper and lower plateau stresses). Tubes were then laser cut into diamonds, stress relieved, and expanded in two steps to their final shape shown in Fig. 2 of Ref. [1]. Parameters used to shape set diamonds are also provided in their publication. Finished diamonds exhibited an $A_{\mathrm{f}}=20 \pm 3{ }^{\circ} \mathrm{C}$ as determined by the bend and free recovery method defined in ASTM F2082-06. Electropolishing diamonds targeted a total weight loss of $25 \%$ to ensure uniform surface smoothness and eliminate heat-affected zones from laser cutting operations.

\section{Material Impurities}

As occurs in most metal casting processes, undesirable residual elements form non-metallic inclusions (NMI) that can affect fatigue properties of a final component. In Nitinol, as with other structural alloy systems, interstitial elements such as $\mathrm{C}$ and $\mathrm{O}$ form NMI of the general stoichiometry $\mathrm{Ni}_{x} \mathrm{Ti}_{y} \mathrm{O}_{z} \mathrm{C}_{w}$, with $\mathrm{O}$ often reported as $\mathrm{O}+\mathrm{N}$, existing as oxides, carbides, or combinations thereof. As a result of severe reductions required to process a cast ingot through final tubing, brittle NMI particles fracture with localized plastic flow of the matrix causing debonding of particle and matrix and creation of a void between the two. The axisymmetric nature of the drawing process elongates voids creating particle-void assemblies (PVA) as defined by Rahim et al. [16]. Depending on specific manufacturing processes, continued reductions may close elongated voids partially healing the matrix around a particle. Continued drawing can result in repeated particle fracture, void formation, elongation, and closure creating a series of linear defects known as inclusion-cluster 'stringers.' Such defects often act as crack nucleation sites in high-cycle fatigue [17]. As discussed later, the extent of void formation, closure, and rebonding between particle and matrix may have a significant effect on fatigue behavior of a final component.

Knowing the important role inclusions play in high-cycle fatigue life, there is a desire to predict the fatigue life of a Nitinol component based on measured levels of residual elements or inclusions in the as-received mill product as reported in the certificate of compliance ( $\mathrm{C}$ of $\mathrm{C})$. Such information is provided in Table 1 for the two ingots in the current work as well as the five ingots from the Ref. [1] study. Typically, Nitinol made using only a VAR process tends to exhibit very low $\mathrm{C}$ content as is seen for the Standard VAR and HP-VAR ingots with levels less than 20 ppmw. In contrast, Table 1 shows the materials made using a VIM or combination VIM-VAR process exhibit greater C levels of 268, 269, and 330 ppmw for the Standard VIM-VAR, PO VIM-VAR, and Standard VIM materials, respectively [1]. The $\mathrm{C}$ levels seen in the two Standard VIM-VAR ingots used in the current study, i.e., 272 and 283 ppmw for Ingots 1 and 2, respectively, are comparable to the two VIM-VAR ingots in the Ref. [1] study. At 330 ppmw, the C level in the Standard VIM material is about $23 \%$ greater than in the VIM-VAR materials in either study. Other than the HP-VAR material, no correlation was found between fatigue life and $\mathrm{C}$ content as reported in the $\mathrm{C}$ of $\mathrm{C}$ by the mills in either the earlier [1] or the current work.

In the case of $\mathrm{O}$ (reported as $\mathrm{O}+\mathrm{N}$ ), the HP-VAR exhibits substantially less $\mathrm{O}+\mathrm{N}$ at 63 ppmw compared with the Ref. [1] Standard VAR, the Standard VIM-VAR, and the PO VIM-VAR that contained 274, 197, and 260 ppmw, respectively. The Standard VIM-VAR materials in the current study also exhibited relatively high $\mathrm{O}+\mathrm{N}$ levels at 157 and 250 ppmw for Ingots 1 and 2, respectively. As with $\mathrm{C}$, the $\mathrm{O}+\mathrm{N}$ level in the Standard VIM material in Ref. [1] was notably greater at 410 ppmw. Rahim et al. [16] reported Nitinol materials with higher $\mathrm{O}$ contents exhibit reduced fatigue life. As for $\mathrm{C}$, other than the HP-VAR material, no correlation was found between fatigue life and $\mathrm{O}+\mathrm{N}$ as reported by the mills in either the previous [1] or current work.

Although difficult to compare directly due to differences in analytical techniques used by different mills, the maximum NMI lengths reported in Table 1 range from $7 \mu \mathrm{m}$ for the Standard VIM material to $38 \mu \mathrm{m}$ for the Standard VAR material [1]. Those for the Standard VIM-VAR ingots in the current study fall within this range at 9 and $15 \mu \mathrm{m}$ for Ingots 1 and 2, respectively. As well, the areal fraction of NMI for the materials in Ref. [1] ranges from 0.46 to $1.25 \%$ for the HP-VAR and Standard VAR, respectively, with those for the two ingots used in the current study comparable at $\approx 0.75 \%$. Other than the Standard VAR material exhibiting the largest NMI length and overall worst fatigue life, there are no apparent correlations between fatigue life and either NMI maximum length or areal fraction as reported by the mills for either study. Ingot transformation temperatures also reported in Table 1 for all materials in both studies range between $-19{ }^{\circ} \mathrm{C}$ for the Standard VAR and $-1{ }^{\circ} \mathrm{C}$ for the Standard VIM in the previous study [1].

\section{Test Methodology}

Longitudinal loading of struts occurring during fatigue testing requires finite element analyses (FEA) to calculate operational stresses and strains and corresponding linear displacements. The commercial FEA package ABAQUS 
(Standard Version $2017^{\circledR}$ ) was used in combination with the user-defined material subroutine (UMAT) Nitinol-3D for these computational analyses. The non-linear mechanical properties for the Nitinol UMAT were derived from uniaxial tension tests conducted in accordance with ASTM F2516 [18] using the same Nitinol tubes with identical thermal treatments as those used to shape set diamondshaped specimens. Tensile data are presented in Table 2 for the current study as well as from Ref. [1]. The displacements required to reach the desired pre-strain of $6 \%$ and mean strain of $3 \%$ were computed at cycle 1 , whereas the displacement inputs to achieve the various strain amplitudes were computed at cycle 3 of the FEA analysis. This technique of utilizing computational modeling to establish experimental displacement inputs is common in the evaluation of Nitinol stent-like surrogates [6, 19-22].

Fatigue testing was performed using 12-station BOSE Electroforce Model 3200 mechanical testing units. Testing was performed in displacement control with specimens submersed in a phosphate-buffered saline (PBS) solution at $37 \pm 1{ }^{\circ} \mathrm{C}$ at frequencies ranging from 20 to $60 \mathrm{~Hz}$ depending on displacements so as to remain within operating conditions prescribed by the manufacturer. Samples were prestrained to $6 \%$ then unloaded to $3 \%$ strain to simulate catheter loading and vascular deployment. A minimum of six samples were cycled at each condition ranging from 0.5 to $2.9 \%$ strain amplitude. Samples were cycled until fracture or runout at $10^{7}$ cycles. Individual load cells monitored loads on each sample and served as break detection. A total of 177 samples were tested with 70 fractures occurring and 107 surviving to the runout condition. All fractures occurred in the high-stress volume region of the strut.

\section{Results}

Diamond fatigue data for tubing processed using TM-1 are shown in Fig. 2 on a standard strain-life plot with strain amplitude, $\varepsilon_{\mathrm{a}}$, plotted as a function of number of cycles to fracture, $N_{\mathrm{f}}$, for tube lots TM-1: $1-1$ (filled red circles), 1-2 (open red circles), and 2-1 (filled green circles) made from Standard VIM-VAR Ingots 1 and 2 as outlined in Table 1. Black arrows indicate runout samples. An increasing number of runouts were observed with reduced strain amplitudes. Runouts were observed at all strain amplitudes except at $2.9 \%$, where all samples fractured in the low-cycle region $N_{\mathrm{f}} \leq 10^{5}$ cycles. No fractures occurred below $1.3 \%$ strain amplitude. Immediately apparent in Fig. 2 is the dearth of fractures in the high-cycle fatigue region, i.e., for $N_{\mathrm{f}} \geq 10^{5}$ cycles. This is likely the result of the relatively small volume of highly stressed material during fatigue testing of diamonds [23]. The estimated stressed volume, $\mathrm{V}_{\sigma}$, in a strut based on $10 \%$ of the peak strain amplitude is $\sim 7 \times 10^{-4} \mathrm{~mm}^{3}$ for the diamonds used in this study.

This same trend is observed in the previous diamond fatigue data shown in Fig. 3 for the five materials tested [1] as listed in Table 1. Their diamond fatigue data are shown above the horizontal black dashed line in this figure. In the previous study [1], tension-tension fatigue testing was also performed using 0.229-mm-diameter Nitinol wires made from the Standard VAR, Standard VIM-VAR, and HPVAR materials under the same conditions as the diamonds albeit with a slightly reduced $A_{\mathrm{f}}=17 \pm 3{ }^{\circ} \mathrm{C}$. The gauge length of their wire was $101.6 \mathrm{~mm}$ resulting in a stressed volume $V_{\sigma}=4.2 \mathrm{~mm}^{3}$ or more than $10^{3}$ times that (estimated) of the diamonds used in either study. Their wire
Fig. 2 Strain-life plot for tubing lots $1-1,1-2$, and $2-1$ made using tube manufacturing process TM-1 and fatigued at $37{ }^{\circ} \mathrm{C}$ to the runout condition of $N_{\mathrm{f}}=10^{7}$ cycles. Note the sparsity of fractures occurring in the high-cycle region, i.e., $\sim 10^{5}$ cycles $<N_{\mathrm{f}}<10^{7}$ cycles. See text for discussion

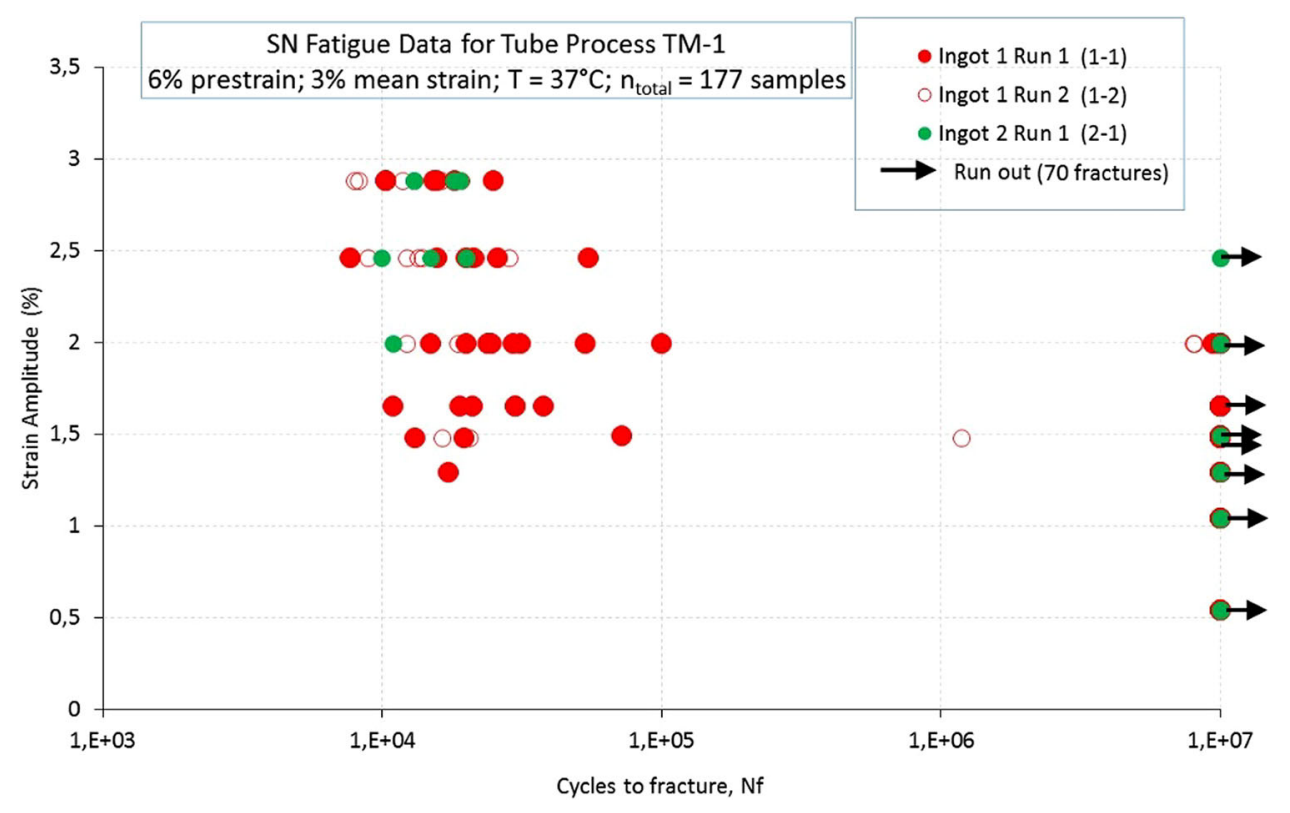


Table 2 Tensile properties of three lots of tubing 1-1,1-2, and 2-1 from the current study used as constitutive input for finite-element analyses

\begin{tabular}{|c|c|c|c|c|c|c|}
\hline Study & Tube lot & Perm. set, $\%$ & Upper plateau, MPa & Lower plateau, $\mathrm{MPa}$ & UTS, MPa & Strain to fracture, $\%$ \\
\hline \multirow[t]{3}{*}{ Current } & $1-1$ & $\approx 0.00$ & 408 & 126 & 1145 & 44.8 \\
\hline & $1-2$ & 0.02 & 399 & 122 & 1138 & 45.0 \\
\hline & $2-1$ & 0.14 & 414 & 138 & 1144 & 43.7 \\
\hline \multirow[t]{5}{*}{ Robertson et al. [1] } & Standard VAR & $\approx 0.00$ & 438 & 188 & NR & NR \\
\hline & Standard VIM-VAR & $\approx 0.00$ & 420 & 170 & NR & NR \\
\hline & Standard VIM & $\approx 0.00$ & 400 & 180 & NR & NR \\
\hline & PO VIM-VAR & $\approx 0.00$ & 415 & 170 & NR & NR \\
\hline & HP-VAR & $\approx 0.00$ & 420 & 188 & NR & NR \\
\hline
\end{tabular}

Estimated tensile data from Ref. [1]. Testing performed per ASTM F2516 [18]

Fig. 3 Strain-life plot for fatigue data from Ref. [1]. Diamond fatigue data are shown above the black dashed line and wire fatigue data below the black dashed line. Note the sparsity of fractures occurring in the high-cycle region, i.e., $\sim 10^{5}$ cycles $<N_{\mathrm{f}}<10^{7}$ cycles for the diamond data. Significantly more high-cycle fractures are observed in the wire data

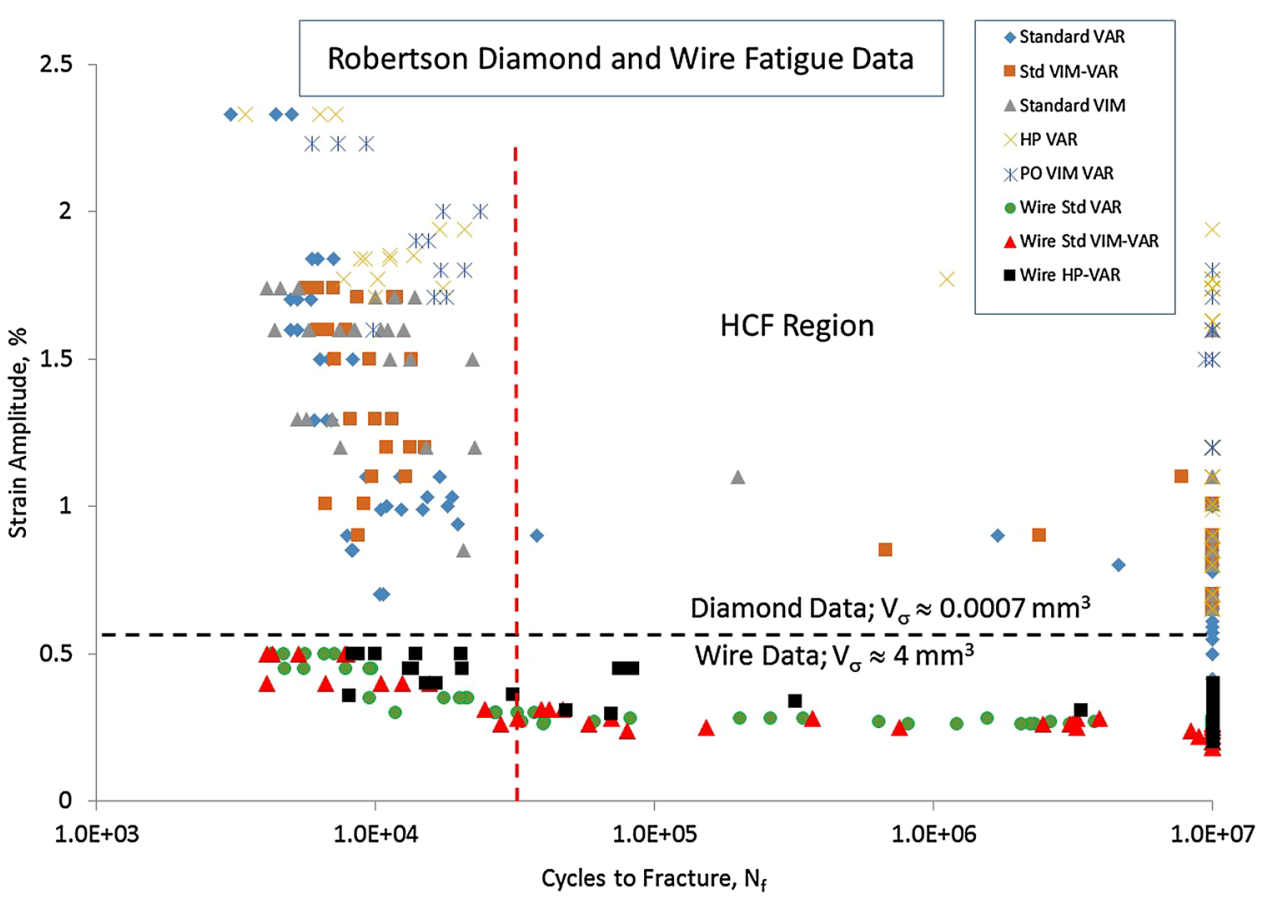

fatigue data are shown below the black dashed line. There are two noteworthy features of these data:

a. There is a plethora of wire fractures in the high-cycle fatigue region compared to the diamond data in both the current study (Fig. 2) and the previous study [1] shown in Fig. 3.

b. There is a considerable reduction in the high-cycle fatigue $(\mathrm{HCF})$ limit of all three materials tested in the wire configuration compared to diamonds. Exemplary is the marked decrease in HCF limit of the HP-VAR material from a value approaching $2 \%$ for diamonds (yellow X's) to less than $0.5 \%$ strain amplitude for wire (filled black squares) in Fig. 3.

These phenomena are likely the result of the significantly larger volume of stressed material in the wires during fatigue testing compared to that in the diamonds. The large $V_{\sigma}$ provides a statistical basis for activating the intrinsic distribution of highest potency crack nucleation sites resulting from normal processing [23]. In contrast, the probability of incorporating a highly potent crack-nucleant in the relatively small volume of highly stressed material in a diamond strut is significantly reduced resulting in:

(a) The typical nearly bimodal distribution of fatigue fractures observed in diamond (or apex) testing of Nitinol (or other structural materials in which $V_{\sigma}$ is relatively small): diamond-type samples tend to exhibit either low-cycle fatigue fractures or runouts.

(b) An increased high-cycle fatigue limit-fewer potent defects result in higher driving forces (stresses or strains) necessary to activate less potent sites and nucleate a fatigue crack. 
As a note, that the HCF limit of the HP-VAR material in wire form (filled black squares in Fig. 3) approaches those for the standard grades of Nitinol at the runout condition is thought to be indicative of a similar distribution of the highest potency nucleating defects in all three materials. The $V_{\sigma}$ in the wire sample is sufficiently large to incorporate and activate these most potent, although sparse, defects in the HP-VAR material. Improvement of the lowprobability fatigue life, important in Class 3 medical devices, is likely best achieved by reducing the maximum size defect in production material as defect size is strongly related to potency in nucleating a fatigue crack.

\section{Microstructural Analyses}

Extensive statistical microanalyses were performed on highly polished longitudinal and transverse sections of finished TM-1 tubing and finished diamond struts made from this tubing. Samples cut from starting hollows used to make these tubes were also examined. Scanning electron (FEI Versa Dual Beam) and optical microscopy (Leica $\mathrm{DMi}^{\mathrm{TM}}$ Metallograph) were performed on all prepared samples. No discernible differences in detecting inclusions were observed between the two imaging modalities. Images were taken with a resolution of approximately $100 \mathrm{~nm} /$ pixel across all imaging methods corresponding to a particle detection limit of $107.9 \mathrm{~nm}$. A minimum of 40 fields of view were taken per surface corresponding to a surveyed area of about $1.5 \mathrm{~mm}^{2}$. Images were analyzed using an automated MATLAB ${ }^{\mathrm{TM}}$ threshold script to detect inclusions/porosity based on image contrast with information exported to Excel files.

Figures $4 \mathrm{a}-\mathrm{d}$ show representative micrographs of longitudinal and transverse sections of finished tubing for lots TM-1: Standard VIM-VAR; 1-1, 1-2 (not shown), and $2-1$. Results of statistical analyses of non-metallic inclusions (NMI) for these three lots are provided in Table 3 for both longitudinal and transverse sections and include maximum, mean, and median NMI lengths; total NMI area fraction plus porosity; NMI density $(\rho)$; average grain size, and area surveyed. Inclusions were analyzed using energydispersive spectroscopy (EDS) and found to be stoichiometric variations of the form $\mathrm{Ti}_{x} \mathrm{Ni}_{y} \mathrm{O}_{z} \mathrm{C}_{w}$ existing as oxides, carbides, or a combination thereof. Table 4 lists similar information for longitudinal sections as reported earlier [1]. It is difficult to directly compare dimensional data from the current study to that from the earlier work as different sample preparation techniques, operators, and computational methods were used in the analyses. Fracture surfaces were examined using optical and scanning electron microscopy (SEM) techniques. All fractures in the current study were found to initiate at an NMI inclusion particle.
In this study, the effects of tube manufacturing processes TM-1 and TM-2 on the fatigue life of a diamond-shaped test surrogate are compared with the intent of correlating such results to underlying microstructural differences that may be attributable to the differences in the two process techniques. The sources of fatigue data for these comparisons are from diamonds made from TM-1 tubing in the current study, and from diamonds made from TM-2 tubing in Ref. [1]. It is desired to correlate both longitudinal and transverse microstructural data to these fatigue results. As described, such microstructural analyses were performed on samples of finished diamonds and final tubing for the TM-1 process. In the case of the previous study [1], however, published microstructural data are from longitudinally sectioned samples only with no transverse data reported or available.

An estimation of microstructural characteristics in transverse direction of the materials studied in Ref. [1] work was made by analyzing two additional tube samples provided by Rau GmbH. The samples were manufactured from a Standard VAR ingot and a Standard VIM-VAR ingot. Although not part of the earlier study per se, these ingots are the same grade and produced by the same mill as Ingots A and B, respectively, used in the earlier study as listed in Table 1. Starting hollows from these two ingots were processed using TM-2 techniques to the same size final tubing used in the Ref. [1] study viz., $8 \times 0.37 \mathrm{~mm}$. These samples, henceforth termed TM-2G Standard VAR and TM-2G Standard VIM-VAR, were analyzed herein solely to gain insight as to relationships between longitudinal and transverse microstructural features of tubing processed using TM-2 techniques. These findings along with similar data from longitudinal and transverse sections of TM-1 samples allowed estimation of the transverse microstructural features of TM-2 processed tubing published in the earlier study. These data were then correlated with fatigue results from both studies as described in the "Discussion" section.

Figure 5a-d are representative micrographs of longitudinal and transverse sections of the TM-2G Standard VAR and TM-2G Standard VIM-VAR samples analyzed using the same techniques as TM-1 samples. Table 5 provides results of statistical analyses of non-metallic inclusions (NMI) for these two samples for both longitudinal and transverse sections.

Figures 4a, c and 5a, c show the microstructural anisotropies resulting from axisymmetric drawing operations typically revealed as stringer-like defects seen in longitudinal sections. In contrast, no microstructural anisotropies are seen in the transverse sections (Figs. 4b, d, 5b, d) as stringers are viewed end-on. NMI dimensional data for transverse sections, although listed as lengths, L, in Tables 3 and 5, are thus more accurately described in terms 


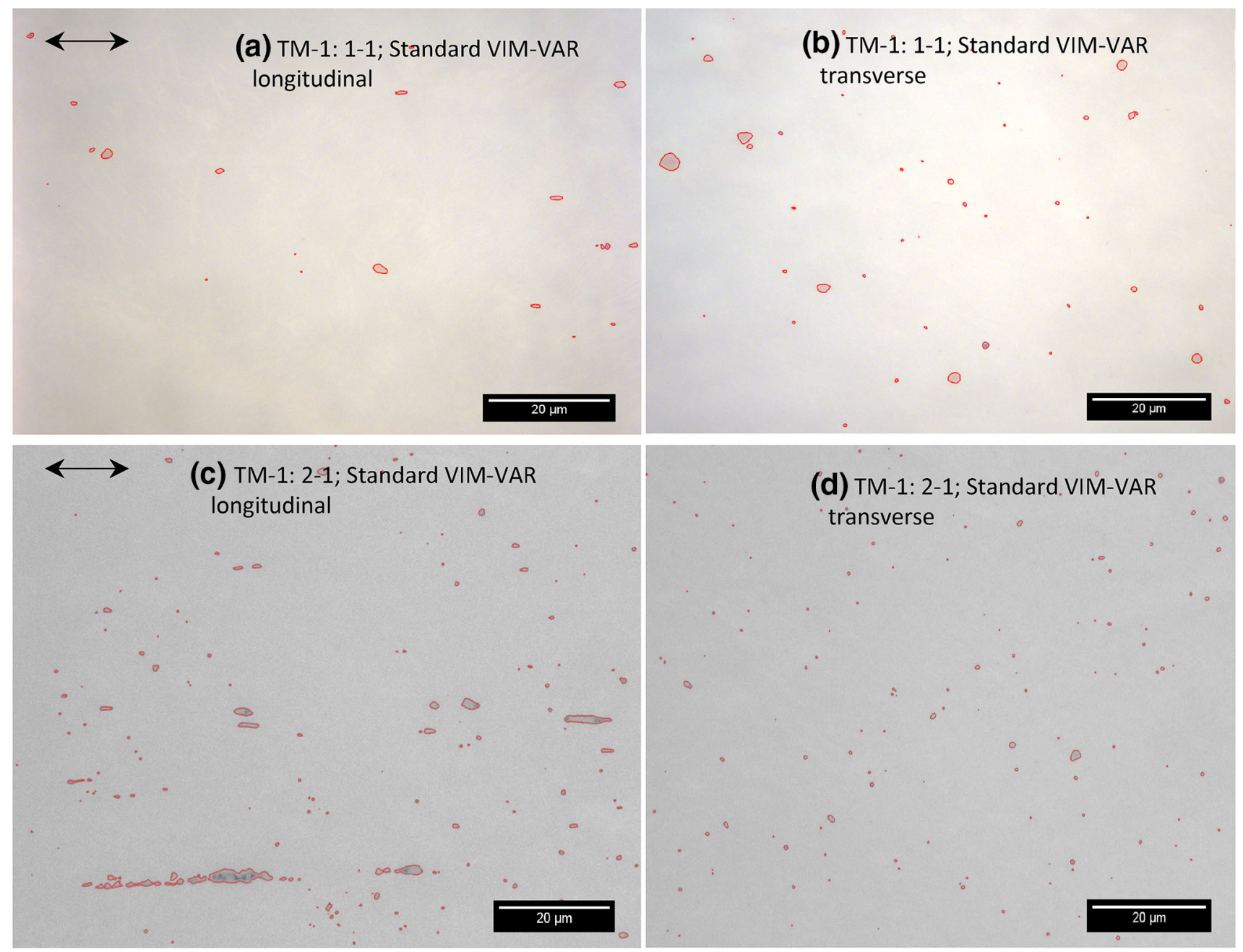

Fig. 4 Representative optical micrographs of finished tubing TM-1: Standard VIM-VAR; a tube 1-1 longitudinal and b tube 1-1 transverse, and $\mathbf{c}$ tube 2-1 longitudinal and $\mathbf{d}$ tube $2-1$ transverse. Tube $1-2$ not shown. Black arrows indicate tube drawing direction

of an effective diameter, $d$, henceforth used to differentiate transverse from longitudinal (length, $L$ ) dimensional information.

Comparison of finished tubing microstructural data to that for starting hollows in Table 3 shows virtually no change in statistical lengths due to drawing operations. Although contrary to [1] who report up to a five-fold increase in maximum NMI length from starting hollow to final tube, the current finding is likely a result of defining an NMI as a contiguous particle/void assembly (PVA) [16] in the current study. Exemplary is the partially healed matrix between two PVA shown in the high magnification BSE image of a longitudinal section of a diamond strut made from TM-1: Standard VIM-VAR; $2-1$ tubing in Fig. 6. Within this definition, partial healing of the matrix through void closure within a single elongated PVA results in two back-to-back PVA with a corresponding decrease in average NMI length. If matrix healing is ignored, the two PVA may be counted as a single NMI unit giving rise to the often observed proximal, but non-contiguous, linear array of particle-void assemblies. Matrix healing was found in TM-1 processed tubing.

Overall, little difference was found between NMI inclusion count and volume fraction of inclusions between the three TM-1 tube lots in longitudinal and transverse sections. Large NMI were found in the TM-2G Standard VAR and TM-2G Standard VIM-VAR tubing samples as listed in Table 5, but not to the size reported by [1] listed in Table 4. In addition to differences in sample preparation and analytical techniques, this may be the result of light etching that tends to increase feature contrast and may result in exaggerated dimensional measurements used in Ref. [1], whereas current samples were polished only. NMI number density, $\rho$ in transverse sections, was found to be 


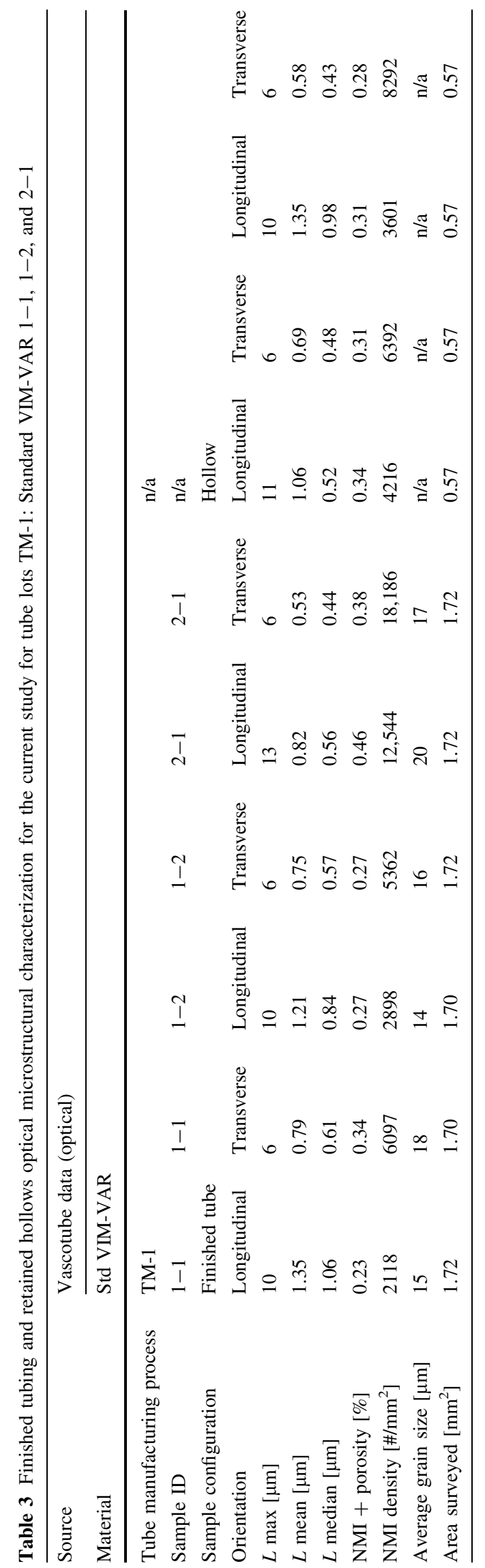

1.5-3 times that in longitudinal sections for all materials examined in the current study. This is simply the result of sectional view and microstructural anisotropy of NMI.

More importantly, Table 3 shows NMI number density $\rho$ for the TM-1: tube Lot $2-1$ is on average 3-5 times greater than that for the other tube lots in this study TM-1: tube Lots $1-1$ and $1-2$. The higher $\rho$ for tube Lot $2-1$ appears to correlate well with reduced mean and median NMI lengths and diameters compared with Lots $1-1$ and 1-2 that show larger dimensions and smaller. These same trends are also seen in data in Tables 4 and 5 for the TM-2 longitudinal data and TM-2G: Standard VIM-VAR, and TM-2G: Standard VAR transverse and longitudinal samples, respectively. While no specific fatigue data exist for the TM-2G samples, phenomenologically, diamonds exhibiting higher fatigue life made using nominal purity raw materials in both studies tend to exhibit higher NMI number densities and reduced NMI dimensions. The exception is the HP-VAR material with very low number density corresponding to the higher-purity raw materials. This follows normal metallurgical tenets that an increased dispersion of finer particles is generally beneficial to mechanical properties [25].

Statistically, Tables 3 and 5 show for longitudinal data:

- $L_{\max }$ : TM-2G: Standard VAR > TM-2G: Standard VIM-VAR > TM-1: $\quad 2-1>$ TM-1: $\quad 1-1=$ TM- 1 : $1-2$.

- $L_{\text {mean }}:$ TM-2G: Standard VAR > TM-2G: Standard VIM-VAR > TM-1: $1-1>$ TM-1: $1-2>$ TM-1: $2-1$.

- $L_{\text {median }}$ : TM-2G: Standard VAR $>$ TM-2G: Standard VIM-VAR $>$ TM-1: $\quad 1-1>$ TM-1: $\quad 1-2>$ TM-1: $2-1$.

And for transverse data:

- $d_{\text {max }}:$ TM-2G: Standard VAR > TM-2: Standard VIMVAR $=$ TM-1: $2-1=$ TM-1: $1-1=$ TM-1: $1-2$.

- $d_{\text {mean }}$ : TM-2G: Standard VAR > TM-1: $1-1>$ TM-1: 1-2 > TM-2G: Standard VIM-VAR > TM-1: 2-1.

- $d_{\text {median }}$ : TM-2G: Standard VAR > TM-1: $1-1>$ TM2G: Standard VIM-VAR > TM-1: 1-2 > TM-1: 2-1.

Examinations of data in Tables 3 and 5 show an approximately linear relationship between $L_{\text {median }}$ and $d_{\text {median }}$ plotted in Fig. 7 with a slope near unity, i.e., $L_{\text {median }}$ $\approx d_{\text {median }}$ for the TM- 1 and TM-2G processed samples. No such relationship is seen for maximum values although mean values exhibited a similar relationship to median values. These NMI dimensional relations are shown to be important aspects to understanding the fatigue lives of materials found in this and the earlier work [1]. 
Table 4 Finished tubing BSE microstructural characterization for samples TM-2: Standard VAR, Standard VIM-VAR, Standard VIM, PO VIMVAR, and HP-VAR tubing from Ref. [1]

\begin{tabular}{|c|c|c|c|c|c|}
\hline \multirow{2}{*}{$\begin{array}{l}\text { Source } \\
\text { Material }\end{array}$} & \multicolumn{5}{|c|}{ Published Robertson Data [1] (BSE) } \\
\hline & Std VAR & Std VIM-VAR & Std VIM & PO VIM-VAR & HP-VAR \\
\hline Tube manufacturing process & TM-2 & & & & \\
\hline Sample ID & $\mathrm{n} / \mathrm{a}$ & & & & \\
\hline Sample configuration & Finished tube & & & & \\
\hline Orientation & Longitudinal & Longitudinal & Longitudinal & Longitudinal & Longitudinal \\
\hline$L \max [\mu \mathrm{m}]$ & 101 & 81 & 50 & 20 & 40 \\
\hline$L$ mean $[\mu \mathrm{m}]$ & 3.55 & 1.86 & 1.29 & 1.40 & 2.88 \\
\hline$L$ median $[\mu \mathrm{m}]$ & 1.72 & 1.23 & 1.06 & 1.06 & 1.26 \\
\hline NMI $[\%]$ & 1.46 & 1.51 & 2.67 & 1.49 & 0.41 \\
\hline Porosity [\%] & 0.12 & 0.05 & 0.05 & 0.01 & 0.02 \\
\hline Total $[\%]$ & 1.58 & 1.55 & 2.72 & 1.50 & 0.43 \\
\hline NMI density $\left[\# / \mathrm{mm}^{2}\right]$ & 3247 & 7272 & 21,483 & 12,702 & 1425 \\
\hline Average grain size $[\mu \mathrm{m}]$ & 14 & 17 & 12 & 14 & 17 \\
\hline Area surveyed $\left[\mathrm{mm}^{2}\right]$ & 0.1 & 0.1 & 0.1 & 0.1 & 0.1 \\
\hline
\end{tabular}

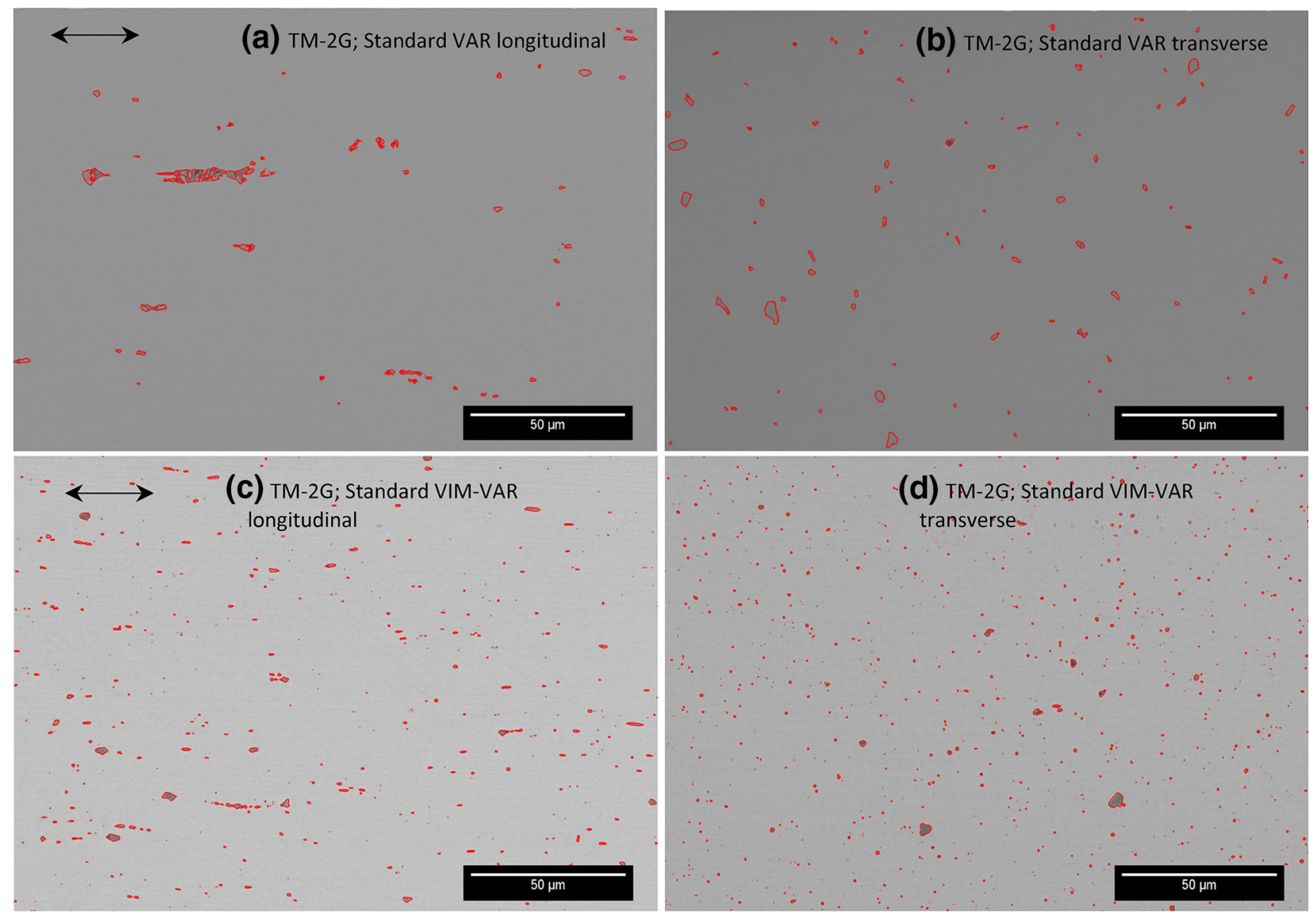

Fig. 5 Representative BSE micrographs of TM-2G samples: a Standard VAR longitudinal, b Standard VAR transverse, c Standard VIM-VAR longitudinal, and d Standard VIM-VAR transverse. Black arrows indicate drawing direction 
Table 5 Finished tubing BSE microstructural characterization for samples TM-2G Standard VAR and TM-2G Standard VIM-VAR
Fig. 6 Representative BSE micrograph of longitudinal section of diamond made from TM-1: Standard VIM-VAR; $2-1$ tubing

\begin{tabular}{lllll}
\hline Source & G. Rau (BSE) & & & \\
\cline { 2 - 5 } Material & Std. VAR & Std. VAR & Std. VIM-VAR & Std. VIM-VAR \\
\hline Tube manufacturing process & TM-2 & & & \\
Sample ID & TM-2G & & & \\
Sample configuration & Finished tube & & & \\
Orientation & Longitudinal & Transverse & Longitudinal & Transverse \\
Lmax $[\mu \mathrm{m}]$ & 40 & 17 & 24 & 6 \\
Lmean $[\mu \mathrm{m}]$ & 2.42 & 1.69 & 1.37 & 0.69 \\
Lmedian $[\mu \mathrm{m}]$ & 1.63 & 1.35 & 1.11 & 0.60 \\
NMI + porosity $[\%]$ & 0.56 & 0.60 & 0.45 & 0.45 \\
NMI density $\left[\# / \mathrm{mm}^{2}\right]$ & 2573 & 4013 & 7393 & 14,088 \\
Average grain size $[\mu \mathrm{m}]$ & $\mathrm{n} / \mathrm{a}$ & $\mathrm{n} / \mathrm{a}$ & $\mathrm{n} / \mathrm{a}$ & $\mathrm{n} / \mathrm{a}$ \\
Area surveyed $\left[\mathrm{mm}^{2}\right]$ & 1.14 & 1.14 & 1.14 & 1.14 \\
\hline
\end{tabular}

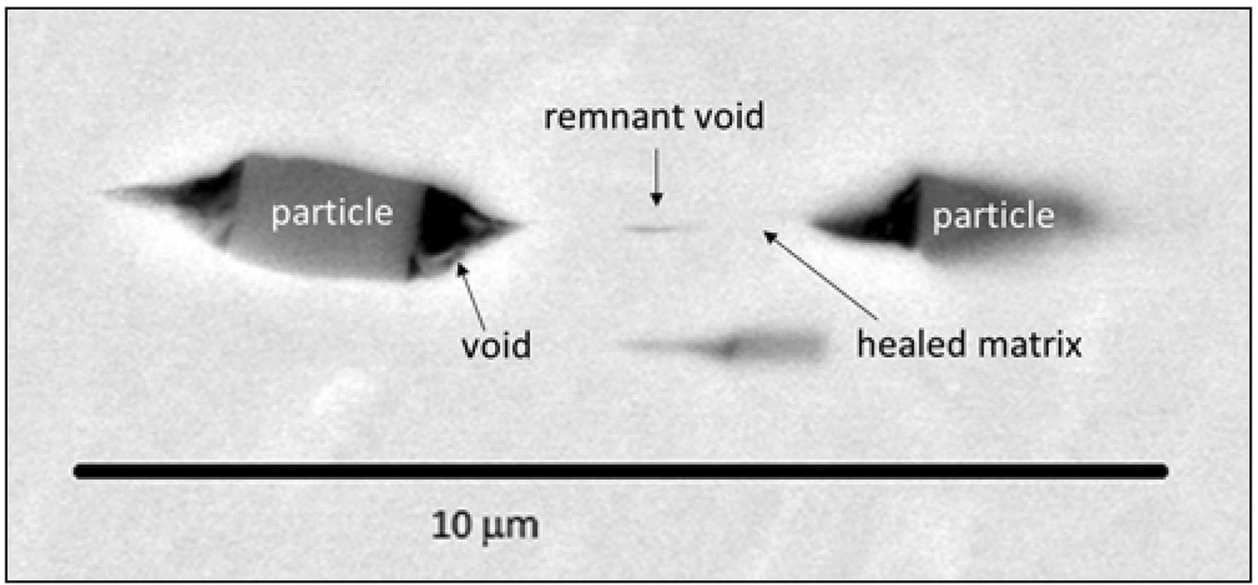

\section{Discussion}

\section{Probabilistic Analyses}

To distinguish trends in fatigue behaviors, strain amplitudes, $\varepsilon_{\mathrm{a}}$, corresponding to 50,5 , and $1 \%$ probabilities of fracture were determined as a function of number of cycles, $N_{\mathrm{f}}$, using the open source program R [26] developed for statistical computing. Output data are in the form of strain amplitudes and number of cycles, $N_{\mathrm{f}}$, for a given probability of fracture. Treating intact diamonds at $10^{7}$ cycles as runouts, the median probabilities, $\mathrm{P}_{\mathrm{LN}}^{50}$, were calculated using a log-normal function while low probabilities, $\mathrm{P}_{\mathrm{W}}^{5}$ and $\mathrm{P}_{\mathrm{W}}^{1}$, were obtained using a 3-parameter Weibull function [27]. The general forms of the cumulative distributions, $\mathrm{F}(\mathrm{x})$, for both functions are given in Eqs. 1 and 2, respectively,

$$
\begin{aligned}
& F(x)=\int_{\lambda}^{x} \frac{1}{\sqrt{2 \pi} \sigma(t-\lambda)} \exp \left[-\frac{[\ln (t-\lambda)-\mu]^{2}}{2 \sigma^{2}}\right] d t, \\
& x>\lambda, \sigma>0
\end{aligned}
$$

$F(x)=1-\exp \left[-\left(\frac{x-\lambda}{\alpha}\right)^{\beta}\right], x \geq \lambda, \alpha>0, \beta>0$

with $\beta$ the shape parameter, $\alpha$ the scale parameter, and $\lambda$ the location parameter. Often a log-normal distribution curve does not accurately represent fatigue data at low probabilities, i.e., as $P \rightarrow 0$. The ability to alter the scale, shape, and location of the curve with the Weibull function allows a better fit and thus a more accurate arithmetic model for these low-probability data.

The fatigue data in the current study shown in Fig. 2 are replotted in Fig. 8 along with the 50\% probability of fracture $\varepsilon_{\mathrm{a}}-N_{\mathrm{f}}$ curves for tube lots TM-1: Standard VIM- 
Fig. 7 Relationship found between $L_{\text {median }}$ and $d_{\text {median }}$ from Tables 3 and 5 for TM-1: Standard VIM-VAR, and TM2G: Standard VAR and TM-2G: Standard VIM-VAR finished tube samples

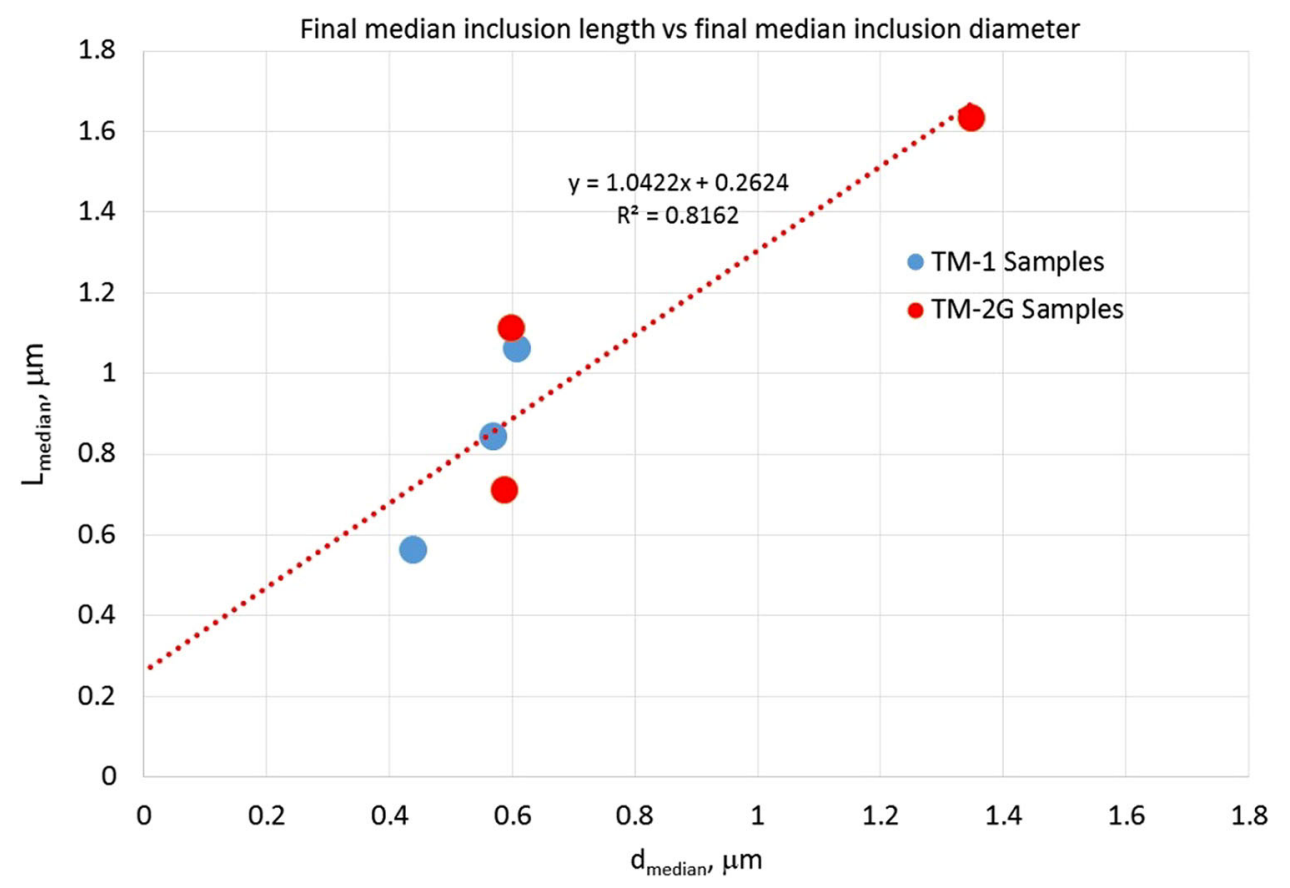

VAR; 1-1 (solid red), 1-2 (dashed red), and 2-1 (solid green). These median curves serve to demonstrate the central tendency of the fatigue behavior of the tube lots [27]. The $P_{\mathrm{LN}}^{50}$ curves for lots $1-1$ and $1-2$ are essentially identical not unexpectedly as both were produced from the same ingot using TM-1 processing. The $P_{\mathrm{LN}}^{50}$ curve for lot $2-1$ is notably different than those for $1-1$ and $1-2$ exhibiting better fatigue behavior in the high-cycle fatigue region as indicated by higher strain amplitudes required to fracture half the samples over the majority of $N_{\mathrm{f}}$.

Also shown in the blue boxes on the right side of Fig. 8 are the strain amplitudes at the runout condition of $10^{7}$ cycles for $P_{\mathrm{LN}}^{50}$ for the three tube lots in the current study. As these strain amplitudes essentially comprise a probabilistic high-cycle $\left(10^{7}\right.$ cycles) fatigue endurance limit (FEL), henceforth, we use these values corresponding to each probability of fracture $P, \varepsilon_{\mathrm{a}}^{P}$, to characterize, and as a basis for comparing, the high-cycle fatigue behavior of all materials examined. Thus, $\varepsilon_{\mathrm{a}}^{50} \approx 1.73 \%$ for tube Lots $1-1$ and $1-2$ while that for tube Lot $2-1$ is about $30 \%$ higher with $\varepsilon_{\mathrm{a}}^{50}=2.25 \%$. Since all three tube lots were processed identically using TM-1, this difference is likely the result of statistical differences in microstructures in the final tubing as will be demonstrated. Table 6 lists $\varepsilon_{\mathrm{a}}^{P}$ at $N_{\mathrm{f}}=10^{7}$ cycles for $P=50,5$ and $1 \%$ for all materials tested.

The $\varepsilon_{\mathrm{a}}^{\mathrm{P}}-N_{\mathrm{f}}$ curves corresponding to 50,5 , and $1 \%$ probabilities of fracture were also determined for the diamond fatigue data shown in Fig. 3 and are compared with those in the current study in Figs. 9, 10, and 11, respectively. No raw data are shown in these figures as the curves represent probabilistic fatigue behaviors of diamonds in both studies.

Of particular interest in these figures is the difference in the probability of fracture curves between tubing made from Standard VIM-VAR Nitinol processed via TM-1, and the five materials produced via TM-2. Despite using a standard grade of Nitinol, diamonds made from TM-1 tubing exhibit markedly superior fatigue lives to those processed using TM-2 for the standard grades of Nitinol, and quite similar fatigue behavior to the cleaner HP-VAR and PO VIM-VAR specifically developed to exhibit superior fatigue performance. Indeed, consistent with [1] findings these cleaner materials do exhibit superior fatigue life to the other materials tested and processed via TM-2 but not when compared with TM-1 results. This difference in properties is likely a direct result of the difference in final microstructures and matrix properties related to differences in tube drawing techniques.

In general then and within confidence intervals, comparison of data presented in Table 6 and Fig. 9 shows for $P=50 \%$ in the high-cycle fatigue region, for $N_{\mathrm{f}}>10^{5}$ cycles:

- TM-1: Standard VIM-VAR; 2-1 exhibits superior fatigue life at all probabilities to all materials and processes.

- TM-1: Standard VIM-VAR; $1-1$ and 1-2 exhibit the second-best fatigue life and are virtually identical.

- TM-2: HP-VAR exhibits the third best fatigue life converging with the TM-1: $1-1$ and $1-2$ curves at $10^{7}$ cycles. 


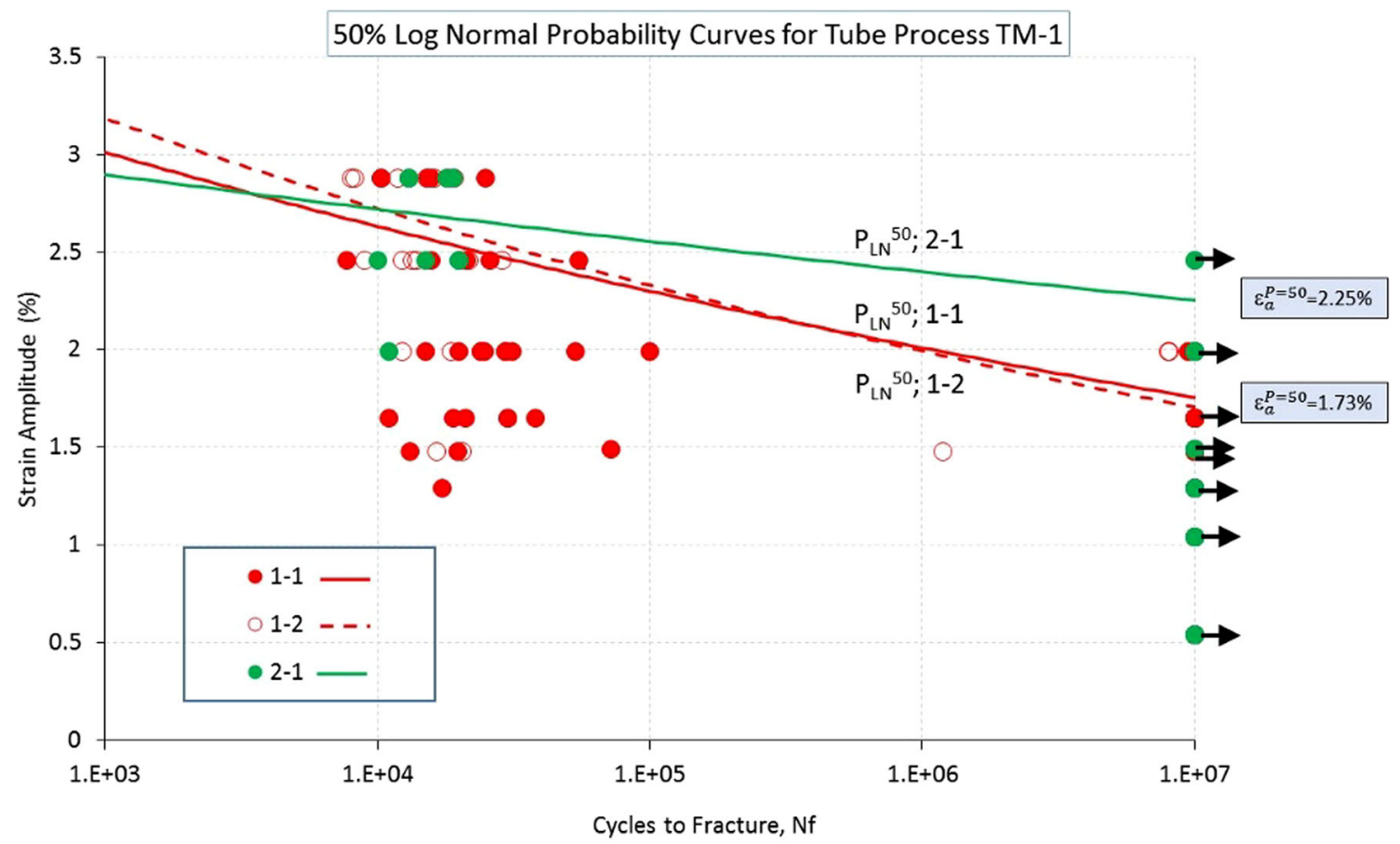

Fig. $8 \varepsilon_{\mathrm{a}}-N_{\mathrm{f}}$ data from Fig. 6 with $\mathrm{P}_{\mathrm{LN}}^{50}$ curves for tube lots $1-1$ (solid red), 1-2 (dashed red), and 2-1 (solid green). $\mathrm{P}_{\mathrm{LN}}^{50}$ for lots $1-1$ and $1-2$ are essentially identical. $\varepsilon_{\mathrm{a}}^{50}$ at $10^{7}$ cycles are also shown

Table $6 \varepsilon_{\mathrm{a}}^{\mathrm{P}}$ for $P=50,5$, and $1 \%$ at $10^{7}$ cycles for the TM- 1 and TM-2 tubing

\begin{tabular}{llcl}
\hline Material & $\varepsilon_{\mathrm{a}}^{50} @ 10^{7}$ cycles, $\%$ & $\varepsilon_{\mathrm{a}}^{5} @ 10^{7}$ cycles, $\%$ & $\varepsilon_{\mathrm{a}}^{1} @ 10^{7}$ cycles, \% \\
\hline TM-1: Standard VIM-VAR; $1-1$ & 1.75 & 1.13 & 0.85 \\
TM-1: Standard VIM-VAR; $1-2$ & 1.71 & 1.19 & 0.92 \\
TM-1: Standard VIM-VAR; $2-1$ & 2.25 & 1.78 & 1.51 \\
TM-2: HP-VAR & 1.71 & 1.33 & 1.11 \\
TM-2: PO VIM-VAR & 1.56 & 1.23 & 1.04 \\
TM-2: Standard VIM & 1.00 & 0.71 & 0.54 \\
TM-2: Standard VIM-VAR & 0.83 & 0.59 & 0.47 \\
TM-2: Standard VAR & 0.61 & 0.34 & 0.22 \\
\hline
\end{tabular}

Italic rows compare HCF behavior of TM-1 and TM-2 for the same nominal grade of Standard VIM-VAR Nitinol
- TM-2: PO VIM-VAR exhibits the fourth best fatigue life and is slightly inferior to the HP-VAR.

- TM-2: Standard VIM, TM-2: Standard VIM-VAR, and TM-2: Standard VAR exhibit increasingly worse fatigue lives, respectively.

For $P=5$ and 1\% data in Table 6 and Figs. 10 and 11 demonstrate that:

- TM-1: Standard VIM-VAR; 2-1 exhibits the best fatigue life.

- TM-1: Standard VIM-VAR; 1-1, TM-1: Standard VIM-VAR; 1-2, TM-2: HP-VAR, and TM-2: PO VIM-VAR, exhibit the second-best fatigue lives.
- TM-2: Standard VIM, TM-2: Standard VIM-VAR, and TM-2: Standard VAR exhibit increasingly worse fatigue lives, respectively.

It is of interest to note that for a given probability the slopes of the $\varepsilon_{\mathrm{a}}^{\mathrm{P}}-N_{\mathrm{f}}$ curves are quite similar in the highcycle fatigue region. The exception being the median probability curves for TM-1: Standard VIM-VAR; 1-1 and 1-2 which exhibit a more negative slope. Differences in slopes for the different materials and processes are seen to diminish with decreased probability with curves becoming nearly parallel over the entire range of $N_{\mathrm{f}}$ at $P=1 \%$. As well, slope magnitudes become less negative with decreased probability. These phenomena are reflective 


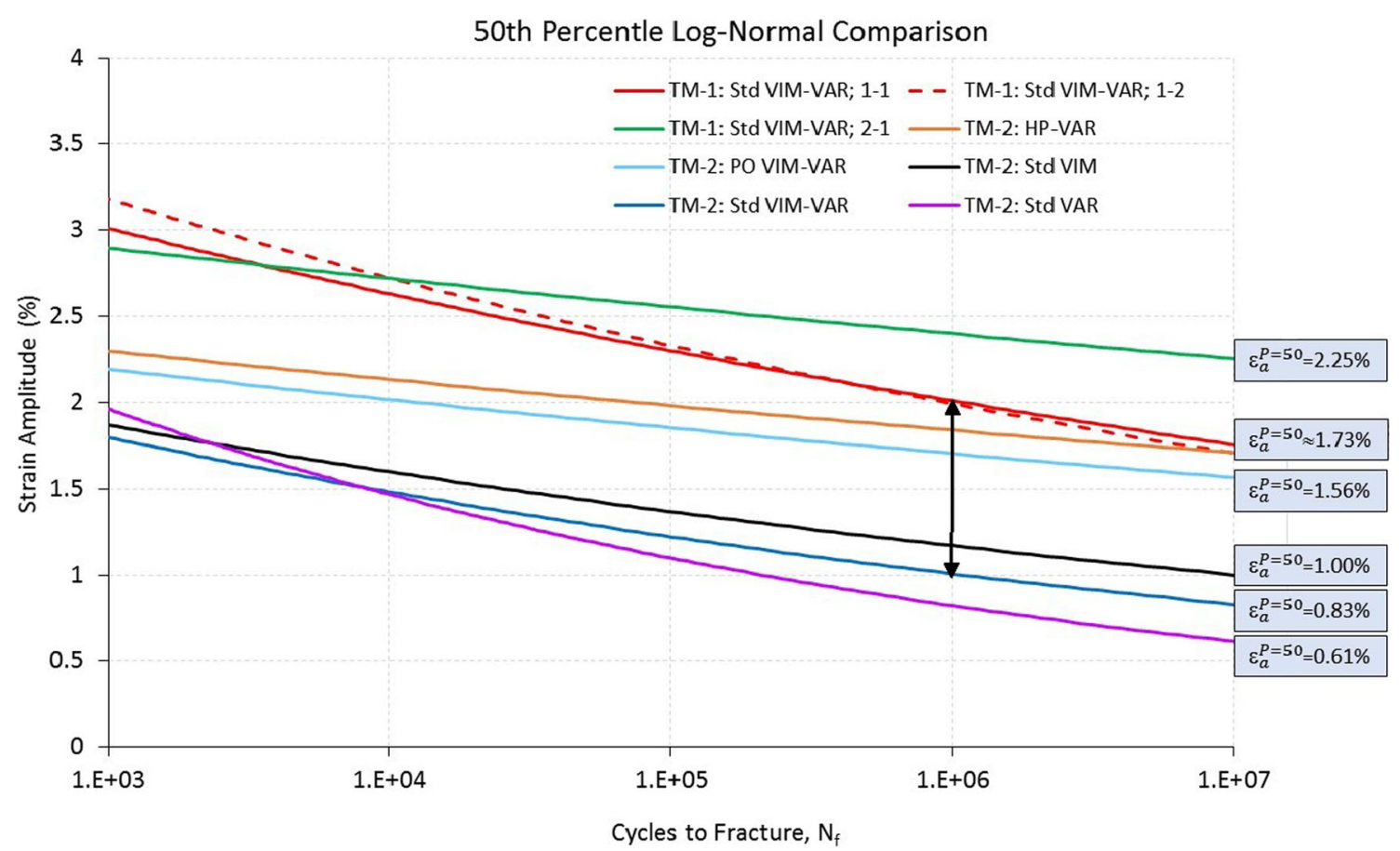

Fig. $9 \varepsilon_{\mathrm{a}}^{50}-N_{\mathrm{f}}$ curves for the TM- 1 data in the current study redrawn from Fig. 8, and fit to the TM-2 diamond data in Fig. 3 from [1]. $\varepsilon_{\mathrm{a}}^{50}$ at $N_{\mathrm{f}}=10^{7}$ cycles for each material is shown in the blue boxes on the

right side of the figure. Vertical double-arrowed black line shows difference between TM-1: Standard VIM-VAR; $1-1$ and $1-2$, and TM-2: Standard VIM-VAR probabilistic fatigue curves

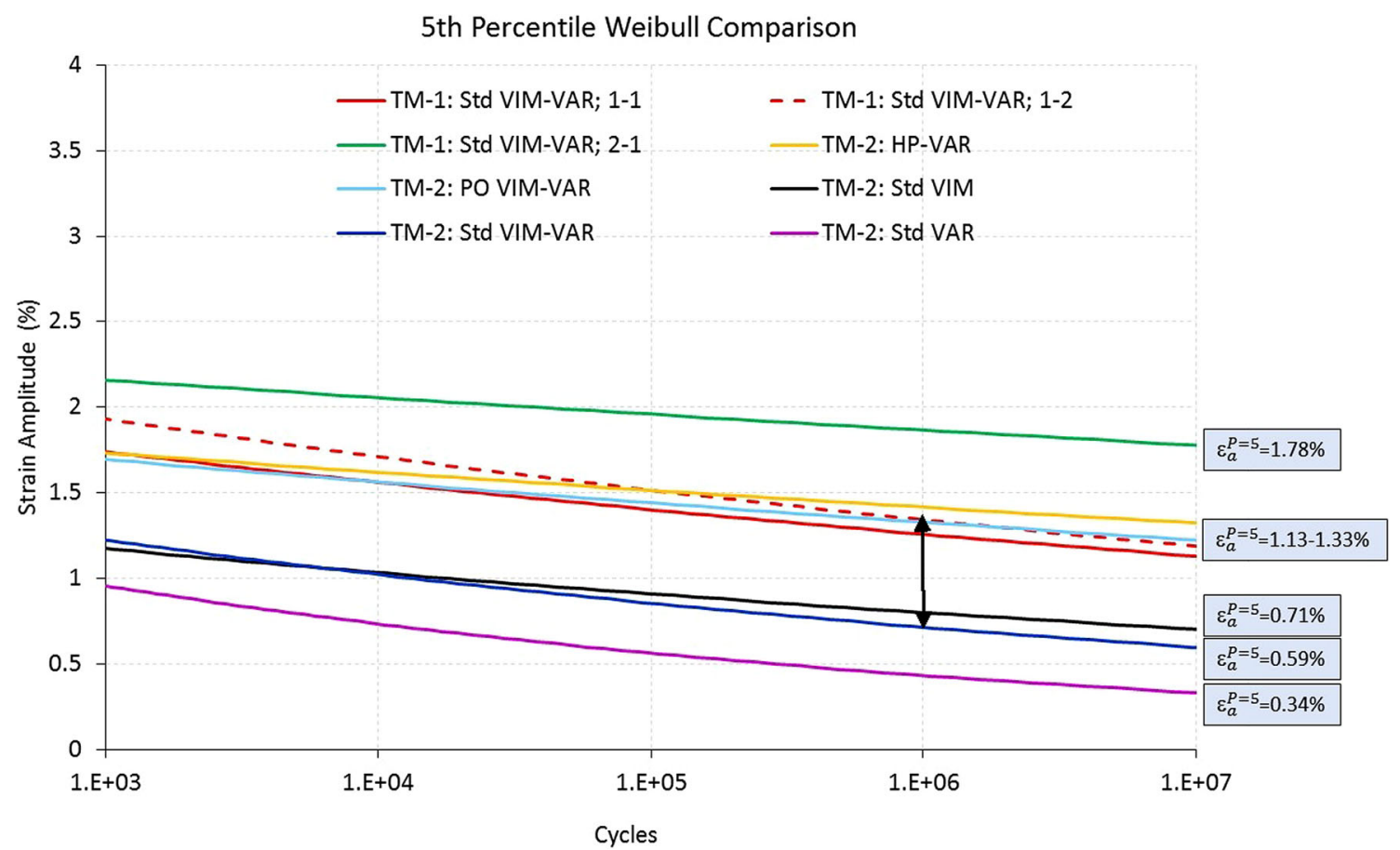

Fig. $10 \varepsilon_{\mathrm{a}}^{5}-N_{\mathrm{f}}$ curves for TM-1 from the current study and TM-2 from Ref. [1]. $\varepsilon_{\mathrm{a}}^{5}$ at $N_{\mathrm{f}}=10^{7}$ cycles for each material is shown in the

black line shows difference between TM-1: Standard VIM-VAR; $1-1$ blue boxes on the right side of the figure. Vertical double-arrowed 
1st Percentile Weibull Comparison

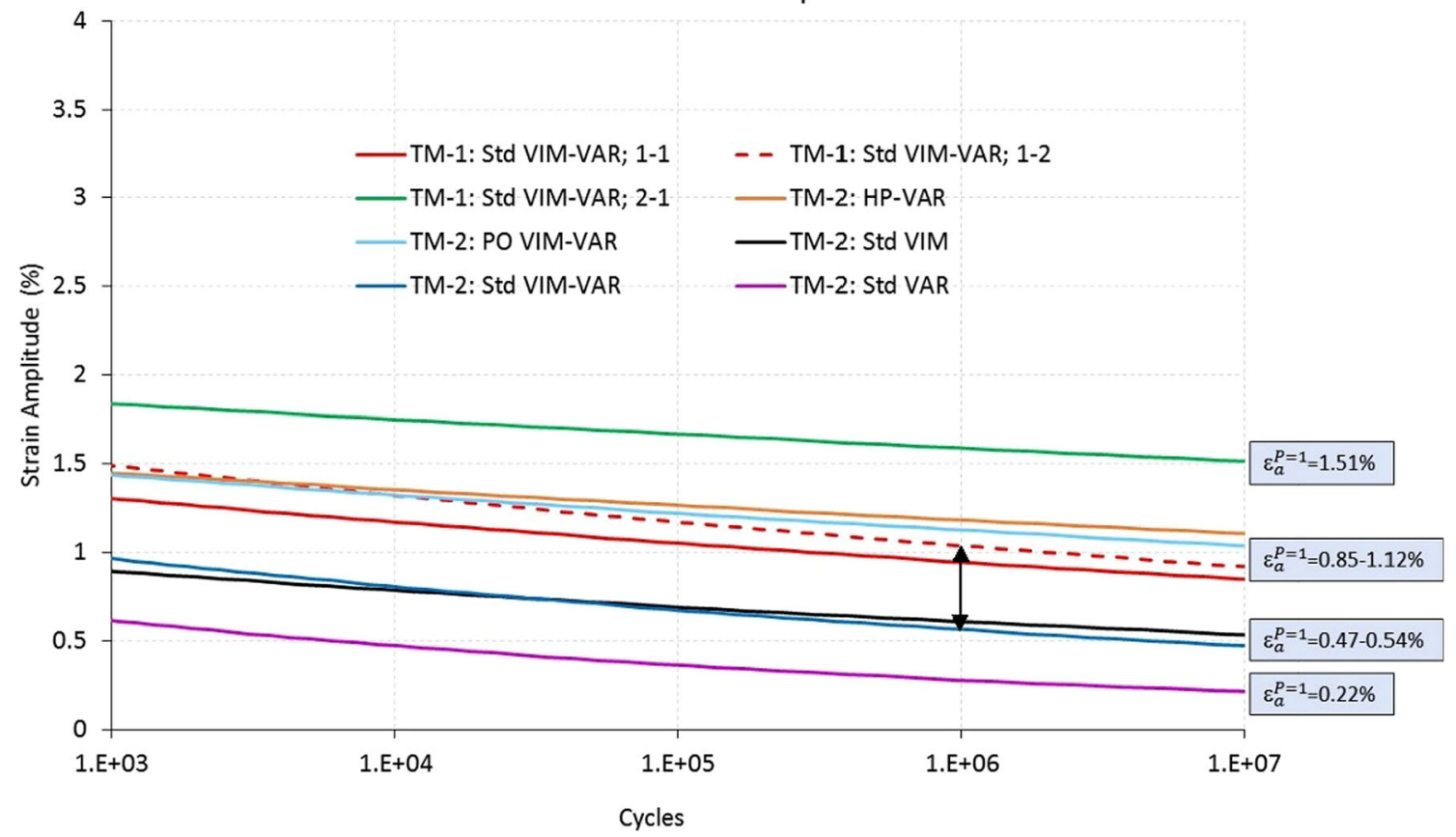

Fig. $11 \varepsilon_{\mathrm{a}}^{1}-N_{\mathrm{f}}$ curves for TM-1 from the current study and TM-2 from [1]. $\varepsilon_{\mathrm{a}}^{1}$ at $N_{\mathrm{f}}=10^{7}$ cycles for each material is shown in the blue boxes on the right side of the figure. Vertical double-arrowed black

of the similarity in shape of the Weibull distribution curves [27] at these extreme values.

\section{Direct Comparison of TM-1 and TM-2}

Robertson et al. [1] tested the five Nitinol materials listed in Table 1 specifically to examine the effects of material cleanliness on fatigue life of diamond surrogates. They ensured all materials were processed the same to minimize the effects of tube processing on results. Since the Standard VIM-VAR Nitinol they used is the same nominal grade and produced by the same raw material supplier as that in the current study, by accounting for differences in starting composition and microstructures between these four tube lots (TM-1: Standard VIM-VAR; $1-1,1-2$, and 2-1, and, TM-2: Standard VIM-VAR), insight into the potential effects of TM-1 and TM-2 tube processing on fatigue behavior may be discerned.

The $\varepsilon_{\mathrm{a}}^{\mathrm{P}}-N_{\mathrm{f}}$ curves for the Standard VIM-VAR Nitinol tubing made using TM-1 and TM-2 are shown in Figs. 9, 10, and 11 for the three probabilities as the solid red line (TM-1: Standard VIM-VAR; 1-1), dashed red line (TM-1: Standard VIM-VAR; 1-2), solid green line (TM-1: Standard VIM-VAR; 2-1), and solid blue line (TM-2: Standard VIM-VAR). Direct comparison of these four curves shows a substantial difference in fatigue behavior between diamonds made from TM- 1 and TM- 2 tubing over the entire line shows difference between TM-1: Standard VIM-VAR; $1-1$ and 1-2, and TM-2: Standard VIM-VAR probabilistic fatigue curves

range of $N_{\mathrm{f}}$ for all probabilities. In the high-cycle fatigue region, $10^{5}$ cycles $<N_{\mathrm{f}}<10^{7}$ cycles, on average the three TM-1: Standard VIM-VAR curves are between 0.5 and $1.5 \%$ higher in absolute strain amplitude than the curve for the TM-2: Standard VIM-VAR. The vertical double-arrowed black lines at the ad hoc abscissa of $N=10^{6}$ cycles in these figures are indicative of the difference between TM-1 and TM-2 probabilistic fatigue curves for the Standard VIM-VAR grade of Nitinol.

For all probabilities, $\varepsilon_{\mathrm{a}}^{\mathrm{P}}$ at $10^{7}$ cycles for the Standard VIM-VAR Nitinol tubing made using TM-1 is about two to three times greater than those for tubing made using TM-2 processing. This difference may be a direct result of the differences in tube manufacturing techniques and related effects on resulting microstructure and matrix properties.

\section{Correlation with Microstructures}

Robertson et al. [1] performed logistic regression analyses on their diamond fatigue data and determined that maximum NMI inclusion length $L_{\max }$ has the largest influence on fatigue resistance compared with other microstructural features. As the minimum probability of fracture is a function of largest defect in a given stressed volume, it is appropriate to plot $\varepsilon_{\mathrm{a}}^{1}(P=1 \%)$ as a function of $L_{\max }$ for the 3 tube lots in the current effort and the five materials from Ref. [1]. These data are plotted in Fig. 12. Over the 
large range of $L_{\max }$ shown in this figure, there is a correlation between $\varepsilon_{\mathrm{a}}^{1}$ and $L_{\max }$ for the TM-2 materials especially at larger values of $L_{\max }$. At the smaller values of $L_{\max }$, the TM-1 data do not exhibit the same relation although values of $\varepsilon_{\mathrm{a}}^{1}$ are approximately the same as for TM-2 despite differences in metallographic sample preparation techniques.

Murakami and Beretta [28] have shown that the fatigue limit of materials containing non-metallic inclusions exhibits a very strong correlation with the nucleating defect size as projected onto a plane perpendicular to the maximum principal stress. They show that small cracks, defects, and non-metallic inclusions having the same 'projected defect size' normal to the maximum principal stress, have identical influence on the fatigue limit regardless of different stress concentration factors. As such, it can be anticipated that the projected defect size will be useful in prediction of fatigue strength.

It would be expected then that the same inclusion can have different effects on fatigue strength depending on the direction of loading and that size and shape of the inclusion are important factors. Thus, different influences of inclusions appear depending on whether loading produces a tensile stress in the longitudinal direction or the transverse direction of an NMI. In the fatigue testing of diamonds, struts are loaded longitudinally, i.e., along NMI axis, and thus NMI length would be expected to have minimal effect on fatigue life of a diamond or stent-like structure subject to pulsatile loading. The reverse would be the case if the struts were loaded in a transverse direction as can occur at the apex of an open-cell poorly designed stent. With these considerations in mind, from a mechanistic perspective, it should be that the projected defect size of an elongated NMI onto a transverse plane of a longitudinally loaded strut might be expected to have a noticeable effect on fatigue life.

Murakami's argument is corroborated by recent finiteelement simulations in which the effects of relative orientations of primary inclusion clusters in steels under fatigue loading were investigated by Salajegheh et al. [29]. Their analyses showed a reduction of nucleation crack potency due to shielding of inclusion pairs when aligned with the applied loading direction. In contrast, they found a marked intensification of potency when pairs were aligned perpendicular to the uniaxial stress direction. This analysis suggests that longitudinal loading of two non-contiguous NMI aligned back-to-back reduces stress intensity but increases it if NMI are aligned side-by-side as is the situation in the transverse plane of a strut.

Adopting the 'projected defect size' model, we use NMI data shown in Table 3 for the TM- 1 tubing in the current work as values suitable for correlating to fatigue lives. Specifically, median diameters, $d_{\text {median}}$, as measured on transverse-sectioned planes of TM-1 processed samples are found to correlate well with median probabilistic $10^{7}$ cycle fatigue endurance limits, $\varepsilon_{\mathrm{a}}^{50}$. Although [1] did not perform microstructural analyses on transverse sections of samples, estimates of $d_{\text {median }}$ for the five materials in their study were obtained from the relationship between $L_{\text {median }}$ and $d_{\text {median }}$ shown in Fig. 7 viz., $L_{\text {median }} \approx 1.04 d_{\text {median }}$, derived from measurements of $L$ and $d$ on tube lots made using TM-1, and the additional TM-2G samples as listed in
Fig. $12 \varepsilon_{\mathrm{a}}^{1}$ as a function of $L_{\max }$ for diamonds made from TM-1 tubing in the current study and TM-2 tubing from [1]

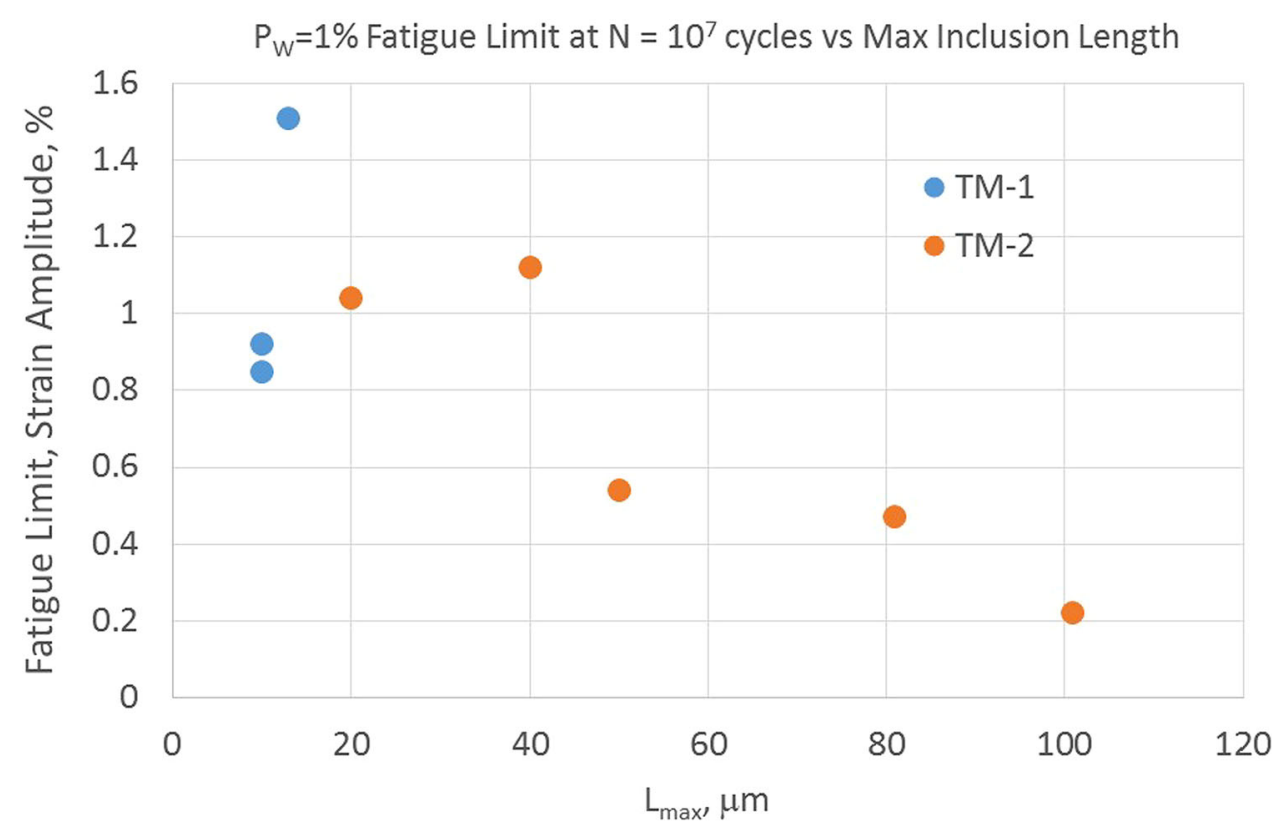


Table 7 Estimated median NMI transverse diameters, $d_{\text {median }}^{\text {est }}$ for all five materials from Ref. [1]

\begin{tabular}{lllllc}
\hline Material & Standard VAR & Standard VIM-VAR & Standard VIM & PO VIM-VAR & HP-VAR \\
\hline $\begin{array}{c}L_{\text {median }}, \\
\begin{array}{c}\mu \mathrm{m} \\
\text { est } \\
\text { median } \\
\mu \mathrm{m}\end{array}\end{array}$ & 1.72 & 1.23 & 1.06 & 1.06 & 1.26 \\
\hline
\end{tabular}

Table 5. Using the $L_{\text {median }}$ data in Table 4 , estimates for $d_{\text {median }}$ are given in Table 7 for all five materials from the [1] study.

Using the data in Tables 3, 6, and 7, Fig. 13 is a plot of $\varepsilon_{\mathrm{a}}^{50}$ vs. $d_{\text {median }}$ for all materials from both studies. A best-fit curve through the six data points for the standard grade materials from both studies, i.e., TM-1: Standard VIMVAR, 1-1, 1-2, and 2-1, and, TM-2: Standard VIM, Standard VIM-VAR, and Standard VAR show these data follow an inverse power-law function with a negative exponent near unity.

The data points for the HP-VAR and PO VIM-VAR materials do not lie on this curve. To fit the curve in Fig. $13, d_{\text {median }}$ would need to be $\sim 0.6 \mu \mathrm{m}$ for the values of $\varepsilon_{\mathrm{a}}^{50}$ determined for these two materials. This value is essentially at the limit of particle detection of $0.59 \mu \mathrm{m}$ reported in the [1] study. This lower bound limit on particulate detection artificially limits the NMI statistical analyses results: to wit, the minimum statistical particle length found in Ref. [1] for any material was $L_{\text {median }}$ of $1.06 \mu \mathrm{m}$, thus limiting the minimal estimated $d_{\text {median }}$ values shown in Fig. 13. With nearly six times greater resolution limit of $107.9 \mathrm{~nm}$ of the microanalytical techniques used in the current study and 10-17 times greater surveyed area, a corresponding minimal statistical particulate size of $d_{\text {median }}=0.44 \mu \mathrm{m}$ was determined for TM-1: Standard VIM-VAR; $2-1$ (Table 3 ), and $d_{\text {median }}=0.60 \mu \mathrm{m}$ in the TM-2G: Standard VIM-VAR tubing (Table 5). It is thus reasonable that analyses of the Ref. [1] samples using techniques employed in the current study would have resulted in reduced dimensional values and the possibility of the two data points for the high-purity materials following observed relationship for the other materials.

The power-law relationship between the median probabilistic fatigue limits, $\varepsilon_{\mathrm{a}}^{50}$, and median NMI transverse diameters $d_{\text {median }}$ for the materials and processes shown in Fig. 13 is consistent with data for steels found by Murakami [23] albeit with a negative exponent of $1 / 6$ or about $1 / 6$ th that found for the Nitinol materials in the current study. The reduced dependence of fatigue life on nucleating defect size in the steels may be related to the much larger defect sizes that ranged from 25 to $1000 \mu \mathrm{m}$ compared to the approximately 1 micron size inclusions in the Nitinol materials.
The data in Fig. 13 comprise the typical relationship between high-cycle fatigue limit, $\sigma_{\mathrm{f}}$, and dimension of the defect (part of the Kitagawa-Takahashi diagram [30]). Expressing the data in Fig. 13 on a log-log plot (not shown), the relation between $\varepsilon_{\mathrm{a}}^{50}$ and $d_{\text {median }}$ :

$\varepsilon_{a}^{50} \cdot d_{\text {median }} \approx 1$

is obtained for the Nitinol materials and processes explored in the current study. Equation (3) provides a potential basis for developing a predictive relationship between starting microstructure, tube manufacturing technique, and probabilistic fatigue strength of a finished component. Clearly, further work is needed to develop fatigue relationships with microstructures evolving from specific manufacturing processes especially at low probabilities critical to Class 3 medical device efficacies.

\section{Particle Size and Matrix Healing}

Comprehensive reviews that emphasize the impact of NMI particles on fatigue crack initiation [31-35] report that crack nucleation almost always originates at a partially debonded particle [17, 34, 35]. Supportive of these observations are a number of recent numerical simulations modeling the effects of inclusion particle-matrix bonding on crack-nucleant potency. Prasannavenkatessan et al. [36] performed micromechanical simulations of fatigue crack nucleation at second-phase particles that were perfectly bonded to the matrix, partially debonded, and cracked. They found a strong propensity for crack nucleation at inclusion clusters and that the crack-nucleant potency of inclusions is greatly increased by pre-existing particlematrix interfacial damage that occurred during primary metal forming operations, i.e., inclusions with partially debonded interfaces are significantly more potent crack nucleants than intact inclusions which, when coupled with intensification arising due to spatial alignment of inclusions in a cluster often serve as primary sources of fatigue cracking and component failure.

Furthermore, Gall et al. [17] determined in their simulations that cracked inclusions that remain bonded do not lead to localized plastic shear strains nearly as great as those of a debonded or a partially debonded-cracked inclusion. The cracked inclusion carries a significant fraction of the local stresses in the broken particle halves after 
Fig. $13 \varepsilon_{\mathrm{a}}^{50}$ as a function of $d_{\text {median }}$ for TM-1 tubing and TM-2 materials from [1]

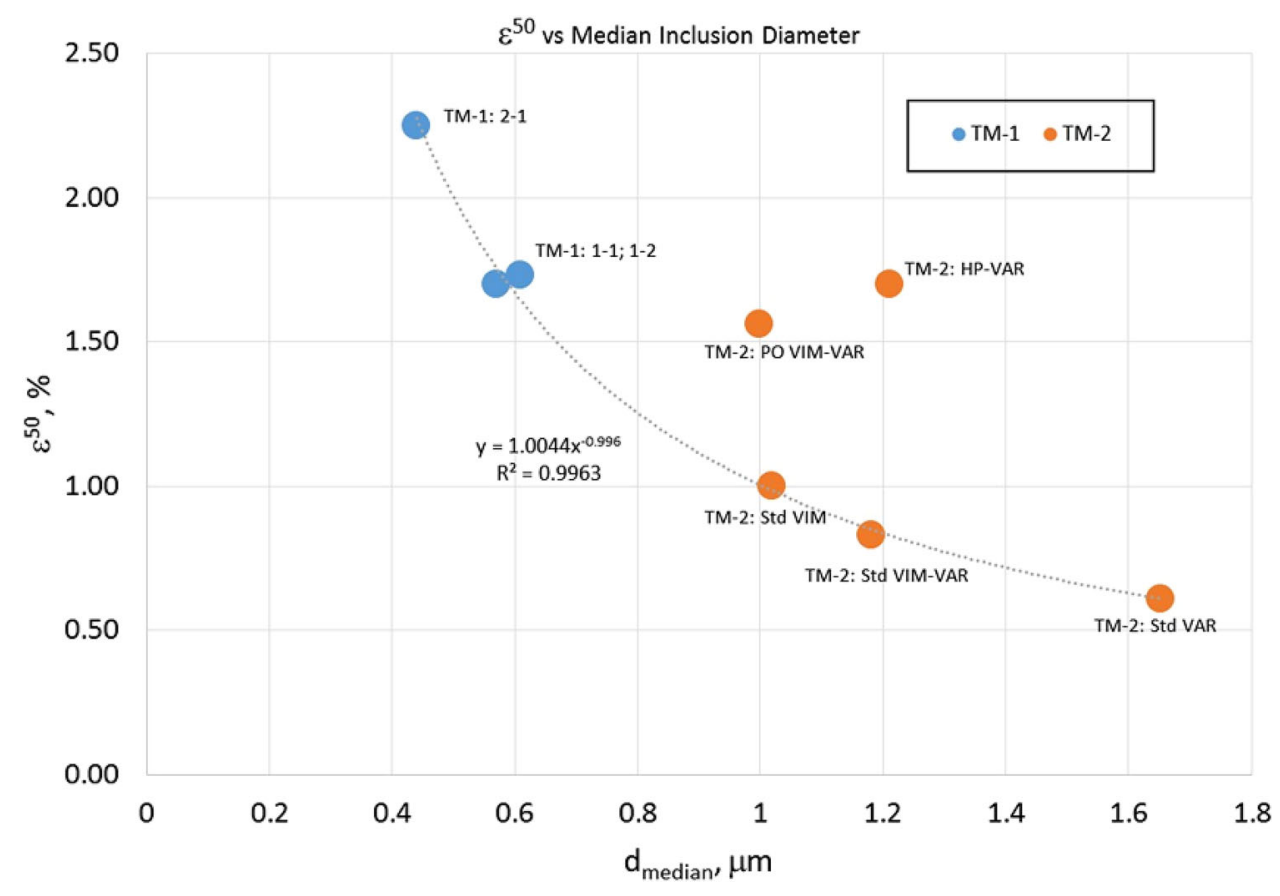

particle fracture, while the debonded inclusion is virtually stress free. Their analyses predict that once a cracked particle begins to debond, the local plastic strains are intensified in the matrix by the same order of magnitude as an un-cracked but partially debonded inclusion.

In the case of Nitinol, simulations of hot deformation of damaged inclusion stringers have shown inter-particle void closure with continued plastic deformation under compressive stress states [37]. These numerical simulations are supported as applications of hot deformation at high pressures to re-bond damaged inclusions have demonstrated significant HCF improvements in bearing steels [38]. The observed relations between higher probabilistic fatigue life and smaller inclusion/void length or diameter in the current study confirm that inclusion and void morphology have notable effects on fatigue life of Nitinol. Based on these observations and the cited importance of particle-matrix bonding on fatigue performance of ductile metals, it is hypothesized that rebonding of damaged particle-matrix interfaces may occur during working of a Nitinol tube with the observed effects on fatigue life of superelastic Nitinol reported herein. The cited role of compressive hydrostatic components of stress on such matrix healing may indicate that unique aspects of TM-1 processes promote such conditions. Further analyses are ongoing.

\section{Conclusions and Future Efforts}

In the present study, we compare the effects of two tube manufacturing techniques, TM-1 and TM-2, on the highcycle fatigue life of three standard grades and two highpurity grades of superelastic Nitinol used in the manufacture of Class 3 cardiovascular medical devices. The results of the current study suggest that tube manufacturing technique may have a significant effect on fatigue life of a finished superelastic Nitinol component. Probabilistic fatigue results were correlated to statistical microanalytical results obtained from samples representative of both the tube manufacturing techniques and compared with the following results:

- For 50, 5, and 1\% probabilities of fracture, the probabilistic fatigue endurance limit (FEL), $\varepsilon_{\mathrm{a}}^{\mathrm{P}}$ at $10^{7}$ cycles, of diamond samples made from the Standard VIM-VAR grade of Nitinol TM-1 tubing is about two to three times greater than $\varepsilon_{\mathrm{a}}^{\mathrm{P}}$ for diamonds made from the same tubing processed using TM-2 techniques [1].

- For all probabilities and despite using a standard grade of Nitinol, diamonds made from TM-1 tubing exhibit markedly superior fatigue performance than different material lots processed using TM-2, and similar fatigue behavior to the cleaner HP-VAR and PO VIM-VAR materials also processed using TM-2.

- For the six tube lots made using standard grades of Nitinol and within the confines of these analyses, median probability fatigue life, $\varepsilon_{\mathrm{a}}^{50}$, exhibits a strong 
dependence on median NMI inclusion transverse diameter following an inverse power-law function with an exponent close to unity consistent with model proposed by Murakami for steels. This type of analysis may provide a basis for development of a predictive relationship between starting hollow microstructure, tube manufacturing technique, and probabilistic fatigue strength of a finished component.

- Based on Murakami's model, and substantiated by FE modeling of primary inclusion clusters, inclusion stringer length per se is expected to have little effect on fatigue life in longitudinal loading conditions as are encountered by the majority of stressed material in a cardiovascular implant.

- Void closures at particle-void assemblies were observed in TM-1 processed samples with concurrent relatively small measured inclusion dimensions. Such closures can facilitate matrix healing and rebonding of the particle-matrix interface with notable improvement of high-cycle fatigue life.

- Future testing and simulations are needed to investigate conjectured attributes of tube processing techniques that may promote matrix healing and reduced NMI size.

The literature cited in this study indicates that the most important effects of NMI on fatigue life in ductile metals are (a) inclusion shape and size, (b) particle-matrix bonding, and (c) relative orientation to applied loads and neighboring inclusions. This study (and others) demonstrates that fatigue of Nitinol is controlled by similar processes and mechanisms as operate in other structural metals despite the unique shape memory aspects of this material.

It is to be noted that the current analyses are applicable to $10^{7}$ cycles only. Estimation of fatigue behaviors to greater number of cycles requires generation of fracture data and related analyses in the range of interest. Development of a more meaningful probabilistic model of highcycle fatigue must utilize either a greater number of test samples or samples with larger stressed volumes such as seen in previous wire data [1]. Such data are the foundation for development of a stressed-volume-based probabilistic fatigue model which when combined with a more mechanistic, less empirical, description of fatigue provides a more accurate basis for predicting device fatigue life to higher $N_{\mathrm{f}}$ and with less need for in vitro testing for device regulatory submissions.

Acknowledgements The authors are grateful to Professors G.B. Olson of Northwestern University, and D.G. Harlow of Lehigh University for discussions regarding fatigue and probability functions. Special acknowledgements are due to Drs. S. Robertson and A. Pelton for their willingness to collaborate on this effort, and Mr. J.F. Boylan for his friendship and long-term mentoring.

Open Access This article is distributed under the terms of the Creative Commons Attribution 4.0 International License (http://crea tivecommons.org/licenses/by/4.0/), which permits unrestricted use, distribution, and reproduction in any medium, provided you give appropriate credit to the original author(s) and the source, provide a link to the Creative Commons license, and indicate if changes were made.

\section{References}

1. Robertson S, Launey M, Shelley O, Ong I, Vien L, Senthilnathan K, Saffari P, Schlegel S, Pelton A (2015) A statistical approach to understand the role of inclusions on the fatigue resistance of superelastic Nitinol wire and tubing. $\mathrm{J}$ Mech Behav Biomater 51:119-131

2. Schulz E, Jabs A, Gori T, Von Bardeleben T, Hink S, KosperKonig W, Vahl CF, Munzel T (2016) Transcatheter aortic valve implantation with the new-generation evolut $\mathrm{R}^{\mathrm{TM}}$ - comparison with corevalve ${ }^{\circledR}$ in a single center cohort. IJC Heart Vasculture 12:52-56

3. Piazza N, Martucci G, Lachapelle K, de Varennes B, Bilodeau L, Buithieu J, Mylotte D (2014) First-in-Human experience with the medtronic corevalve evolut R. EuroIntervention 9:1260-1263

4. Zhu P, Brinson LC, Peraza-Hernandez E, Hartl D, Stebner (2013) A comparison of three-dimensional shape memory alloy constitutive models: finite element analysis of actuation and superelastic responses of a shape memory alloy tube. In: ASME 2013 conference on smart materials, adaptive structures and intelligent systems. Snowbird, Utah

5. Wheeler R, Othmane B, Gao X, Calkins F, Ghanbari Z, Garrison H, Lagoudas D, Petersen A, Pless J, Stebner A, Turner T (2016) Engineering design tools for shape memory alloy actuators: CASMART collaborative best practices and case studies. In: ASME 2016 Conference on smart materials, adaptive structures and intelligent systems, Stowe

6. Pelton AR, Schroeder V, Mitchell MR, Gong XY, Barney M, Robertson SW (2008) Fatigue and durability of nitinol stents. J Mech Behav Biomed Mater 1:153-164

7. Harrison WJ, Lin ZC (2000) The study of nitinol bending fatigue. In: SMST-2000: proceedings of the international conference on shape memory and superelastic technologies. ASM International, Pacific Grove, pp 391-396

8. Pelton AR, Gong XY, Duerig T (2003) Fatigue testing of diamond-shaped specimens. In: Proceedings of the international conference on shape memory and superelastic technologies. ASM International, Pacific, pp 293-302

9. Pelton AR, Pelton SM, Jorn T, Sorg A, Ulmer J, Niedermaier D, Mitchell MR (2017) Fatigue behavior of generation II and generation III nitinol. In: International conference on shape memory and superelastic technologies. ASM International, San Diego

10. Avitzur B (1983) Handbook of metal forming processes; tubing and tubular products. Wiley, Hoboken, p 457

11. ASTM (2012) Standard F2063-12; "Standard specification for wrought nickel-titanium shape memory alloys for medical devices and surgical implants". ASTM International, West Conshohocken

12. Schetky L McD, Wu MH (2003) Issues in the further development of nitinol properties and processing for medical device applications. In: Proceedings ASM materials \& processes for medical devices conference. Anaheim, p 271 
13. Degarmo E, Black JT, Kohser RA (2003) Materials and processes in manufacturing, 9th edn. Wiley, Hoboken

14. Shaheen L (2007) Tube drawing principles: understanding processes, parameters key to quality. In: The tube and pipe journal. The tube \& pipe association, International

15. ASTM Standard F2082-16 (2016) Standard test method for determination of transformation temperature of nickel-titanium alloys by bend and free recover. ASTM International, West Conshohocken

16. Rahim M, Frenzel J, Frotscher M, Pfetzing-Micklich J, Steegmuller R, Wohlschlogel M, Mughrabi H, Eggeler G (2013) Impurity levels and fatigue lives of pseudoelastic NiTi shape memory alloys. Acta Mater 61:3667-3686

17. Gall K, Horstemeyer MF, Degner BW, McDowell DL, Fan J (2001) On the driving force for fatigue crack formation from inclusions and voids in a cast A356 aluminum alloy. Int J Fract 108(3):207-233

18. ASTM Standard F2516-14 (2016) Standard test method for tension testing of nickel-titanium superelastic materials. ASTM International, West Conshohocken

19. Tolomeo D, Davidson S, Santinoranout M (2000) Cyclic properties of superelastic nitinol: design implications. In: Proceedings of the international conference on shape memory and superelastic technologies. ASM International, Pacific Grove, pp 409-417

20. Robertson SW, Ritchie RO (2008) A fracture-mechanics-based approach to fracture control in biomedical devices manufactured from superelastic nitinol tube. J Biomed Mater Res B. 84(1):26-33. http://refhub.elsevier.com/S1751-6161(15)00244-1/ sbref 13

21. Gall K, Tyber J, Wildesanders G Robertson, Robertson SW, Ritchie RO, Maier HJ (2008) Effect of microstructure on the fatigue of hot-rolled and cold-drawn NiTi shape memory alloys. Mater Sci Eng 486:389-403

22. Lin Z, Pike K, Zipse A, Schlun M (2011) Nitinol fatigue investigation on stent-finish specimens using tension-tension method. J Mater Eng Perform 20(4-5):591-596

23. Murakami Y (2002) Metal fatigue: effects of small defects and nonmetallic inclusions. Elsevier Science Ltd., Amsterdam, pp 75-122
24. ASTM Standard F2004-16 (2016) Standard test method for transformation temperature of nickel-titanium alloys by thermal analysis. ASTM International, West Conshohocken

25. Reed-Hill RE (1973) Physical metallurgy principles. 2nd Edn, D. Van Nostrand Co., pp 358-373

26. R Core Team (2013) R: a language and environment for statistical computing. $\mathrm{R}$ foundation for statistical computing, Vienna, ISBN 3-900051-07-0, http://www.R-project.org/

27. Weibull W (1951) A statistical distribution function of wide applicability. J Appl Mech 19(3):293-297

28. Murakami Y, Beretta S (1999) Small defects and inhomogeneities in fatigue strength: experiments, models and statistical implications. Extremes 2(2):123-147

29. Salajegheh N, Prasannavenkatesan R, McDowell DL, Olson GB, Jou H (2014) Finite element simulation of shielding/intensification effects of primary inclusion clusters in high strength steels under fatigue loading. J Eng Mater Tech 136:1-8

30. Kitagawa H, Takahashi S (1976) Applicability of fracture mechanics to very small cracks or the cracks in the early stage. In: Proceeding 2nd international conference mechanical behavior materials, Boston, pp 627-631

31. Suresh S (1998) Fatigue of materials. Cambridge University Press, Cambridge

32. Murakami Y, Endo M (1994) Intl J. Fatigue. 16:163

33. Lukas P (1996) ASM handbook: fatigue and fracture, vol. 19. ASM International Materials Park, p 96

34. Lankford J, Kusenberger F (1973) Met Mat Trans B 4:553

35. J Lankford (1977) Eng Fract Mech 9:617

36. Prasannavenkatesan R, Zhang J, McDowell DL, Olson GB, Jou H-J (2009) 3D modeling of subsurface fatigue crack nucleation potency of primary inclusions in heat treated and shot peened martensitic gear steels. Intl J Fatigue 31:1176-1189

37. Lu C (2017) Private communication

38. Hashimoto K, Fujimatsu T, Tsunekage N, Hiraoka K, Kida K, Santos EC (2011) Effect of inclusion/matrix interface cavities on internal-fracture-type rolling contact fatigue life. Mater Des $32: 4980-4985$ 